\title{
Long Wave Approximations for Water Waves
}

\author{
Jerry L. Bona ${ }^{1}$ Thierry Colin ${ }^{2}$ and David Lannes ${ }^{2}$
}

\begin{abstract}
In this paper, we obtain new nonlinear systems describing the interaction of long water waves in both two and three spatial dimensions. These systems are symmetric and conservative. Rigorous convergence results are provided showing that solutions of the complete free-surface Euler equations tend to associated solutions of these systems as the amplitude becomes small and the wavelength large. Using this result as a tool, a rigorous justification of all the two-dimensional, approximate systems recently put forward and analysed by Bona, Chen and Saut is obtained. In particular, this remark applies to the original system derived by Boussinesq. The estimates for the difference between the Euler variables and the system variables is better than that obtained in the two-dimensional context by Schneider and Wayne who approximated with a decoupled pair of Korteweg - de Vries equations. Indeed, the limitations inherent in approximating by a decoupled system are clarified in our analysis. Results are obtained both on an unbounded domain with solutions that evanesce at infinity as well as for solutions that are spatially periodic.
\end{abstract}

\section{Introduction}

\subsection{Generalities}

The water wave problem for an ideal liquid consists in describing the motion of the free surface and the evolution of the velocity field of a layer of perfect, incompressible, irrotational fluid under the influence of gravity. In this paper, attention is given to both the two-dimensional case wherein the wave motion is presumed not to vary appreciably in one of the coordinate directions, say the $y$-direction in a standard Cartesian coordinate system, and the fully threedimensional setting. However, consideration is restricted to the special case of a flat bottom. It is well understood that several different regimes may be obtained for this problem; attention is given here to the so-called long-wave limit. In this setting, it is presumed that the free surface may be described as the graph of a function $\eta$, say, defined over the bottom. More precisely, the

\footnotetext{
${ }^{1}$ Department. of Mathematics, Statistics, and Computer Science, University of Illinois at Chicago, Chicago, IL,60607, USA bona@math.uic.edu

2 MAB, Université Bordeaux 1 et CNRS UMR 5466, 351 cours de la liberation, 33405

Talence cedex, France colin@math.u-bordeaux1.fr, lannes@math.u-bordeaux1.fr
} 
motion of the fluid is described by the set of equations

$$
\begin{array}{ll}
\frac{h_{0}^{2}}{\lambda^{2}} \Delta \phi+\partial_{z}^{2} \phi=0 & 0 \leq z \leq 1+\frac{a}{h_{0}} \eta(t, X), \\
\partial_{t} \phi+\frac{1}{2}\left(|\nabla \phi|^{2}+\frac{\lambda^{2}}{h_{0}^{2}}\left|\partial_{z} \phi\right|^{2}\right)+\frac{h_{0}}{a} \eta=0 & \text { at } z=1+\frac{a}{h_{0}} \eta(t, X), \\
\partial_{t} \eta+\nabla \phi \cdot \nabla \eta=\frac{\lambda^{2}}{h_{0} a} \partial_{z} \phi & \text { at } z=1+\frac{a}{h_{0}} \eta(t, X), \\
\partial_{z} \phi=0 & \text { at } z=0,
\end{array}
$$

where the operators $\nabla$ and $\Delta$ act on the transverse variable $X \in \mathbb{R}^{d}, d=1$ or 2 . In case $d=1, X=x$ is the coordinate along the primary direction of propagation whilst if $d=2$, then $X=(x, y)$ represents both the horizontal variables. The independent variable $\phi$ is the non-dimensional velocity potential, $z=0$ is the equation of the horizontal and featureless bottom and $z=1+\frac{a}{h_{0}} \eta(t, x, y)$ the equation of the free surface; $a$ is a typical amplitude of the waves, $h_{0}$ the mean depth of the fluid and $\lambda$ a typical wavelength.

The preceding equations are mathematically and numerically recalcitrant. Some results concerning the Cauchy problem wherein the free surface $\eta$ is specified for all values of $X$ and the velocity potential $\phi$ is specified everywhere in the resulting flow domain, both at a given instant of time, are available (see, for example, [11], [17], [19], [21], [22], [16], and the references contained in these works). In many practically important situations, one relies upon simplifications of these equations to describe approximately the behavior of their solutions. Various model equations have been derived by means of formal asymptotic expansions. Historically, the initial developments in this direction were associated with works of Lagrange, St. Venant, Green, Airy and Stokes among others. A very significant step forward was made by Boussinesq [10] who seems to have been the first to properly understand the long-wave regime to be described next.

The long-wave regime is characterized by the presumptions of long wavelength and small amplitude, viz.

$$
\varepsilon=\frac{a}{h_{0}} \ll 1, \quad \frac{\lambda}{h_{0}} \gg 1,
$$

in conjunction with the assumption that the Stokes number

$$
S=\frac{a \lambda^{2}}{h_{0}^{3}}
$$

is of order 1. For the notational simplicity, we take $S=1$ throughout our discussion so that

$$
\frac{\lambda^{2}}{h_{0}^{2}}=\frac{1}{\varepsilon}
$$

If we did not adhere to this presumption, the only change is that the equations would feature the value of $S$ in various of the coefficients. With this notation 
and the presumption that $S=1$, the non-dimensional water wave equations take the form

$$
\begin{array}{rl}
\varepsilon \Delta \phi+\partial_{z}^{2} \phi=0 & 0 \leq z \leq 1+\varepsilon \eta, \\
\partial_{t} \phi+\frac{1}{2}\left(\varepsilon|\nabla \phi|^{2}+\left|\partial_{z} \phi\right|^{2}\right)+\eta=0 & \text { at } z=1+\varepsilon \eta, \\
\partial_{t} \eta+\varepsilon \nabla \phi \cdot \nabla \eta=\frac{1}{\varepsilon} \partial_{z} \phi & \text { at } z=1+\varepsilon \eta, \\
\partial_{z} \phi=0 & \text { at } z=0 .
\end{array}
$$

Perhaps the simplest of the asymptotic models that take account of both nonlinear effects as reflected in the small, but finite amplitude and the dispersive effects coming from large, but finite wavelength is the Korteweg - de Vries equation ( $\mathrm{KdV}$ - equation henceforth) which is a unidirectional, one space dimensional description in terms of the independent variable $\eta$, having the form

$$
\partial_{t} \eta+\partial_{x} \eta+\varepsilon\left(\frac{1}{6} \partial_{x}^{3} \eta+\frac{3}{2} \eta \partial_{x} \eta\right)=0
$$

Note that in the present scaling, the independent variable $\eta$ and its first several partial derivatives are all of order one. This regime has been analysed by Craig [11] starting from the Lagrangian form of (1.1)-(1.4). In terms of the variables introduced above, he showed that there exists a constant $T$ independent of $\varepsilon$ and a solution $\left(\phi^{\varepsilon}, \eta^{\varepsilon}\right)$ of (1.1)-(1.4) defined at least on the time interval $[0, T / \varepsilon]$ such that $\eta^{\varepsilon}$ is approximated to within order $\varepsilon$ in the $L^{\infty}$-norm by an associated solution to (1.5). It is worth noting that according to the formal derivation of the $\mathrm{KdV}$ model as written in (1.5), one expects solutions to be good renditions of an associated Euler flow (1.1)-(1.4) on a time scale of order $\varepsilon^{-1}$ and that neglected effects could make an order one relative contribution on a time scale of order $\varepsilon^{-2}$. Thus Craig's result provides theoretical justification for the use of (1.5), but it has nothing to say about the eventual breakdown of the model as an approximation to (1.1)-(1.4) (see [7], [8], [2] and [1] for more complete discussions of these matters).

Schneider and Wayne [19] extended Craig's result about the KdV-regime by writing a theory that allowed for more general initial disturbances. They also wrote theory for wave motion in both directions. Expressed in the present variables, they showed the solution to (1.1)-(1.4) may be approximated to within order $\varepsilon^{1 / 4}$ on a time scale of order $\varepsilon^{-1}$ by the solutions of two uncoupled, counter-propagating waves, each of which satisfies a KdV-type equation, namely

$$
\left\{\begin{array}{l}
\partial_{t} \eta_{1}+\partial_{x} \eta_{1}+\varepsilon\left(\frac{1}{6} \partial_{x}^{3} \eta_{1}+\frac{3}{4} \partial_{x}\left(\eta_{1}^{2}\right)\right)=0 \\
\partial_{t} \eta_{2}-\partial_{x} \eta_{2}-\varepsilon\left(\frac{1}{6} \partial_{x}^{3} \eta_{2}+\frac{3}{4} \partial_{x}\left(\eta_{2}^{2}\right)\right)=0
\end{array}\right.
$$

While suggestive and interesting as a principle, an error estimate of order $\varepsilon^{1 / 4}$ is clearly not a practically useful bound. A sharp result of this nature appears 
in the present script in Section 5. Similar theory was obtained for a general class of hyperbolic systems by Ben Youssef and Colin in [4]. We will have more to say about this type of approximation presently.

Following the lead of Bona and Smith [9], Bona, Chen and Saut [5] systematically took advantage of the freedom associated with the choice of the velocity variable and made full use of the lower order relations (the wave equation written as a coupled system) in the dispersive terms to put forward a three-parameter family of Boussinesq-type systems, all of which are formally equivalent models of solutions to the two-dimensional Euler equations (1.1)(1.4) when $X=x$. Many of this family were eliminated as potential models when the associated initial-value problems were shown to be ill-posed in [5]. However, there remain significant sub-families that are known to be at least locally well posed in quite reasonable smoothness classes (see [6]). These systems are reviewed in a little more detail in the next section for the reader's convenience.

In this paper, the class of systems developed by Bona, Chen and Saut is extended in an interesting and helpful way. The key to the extensions proposed here is a nonlinear change of variables that leaves the formal order of approximation unchanged, but which results in new systems with very attractive mathematical properties. In particular, we derive systems in both two and three spatial dimensions that are symmetric in their nonlinear structure and their dispersive modelling. An interesting example of the systems we derive and analyse is

$$
\begin{aligned}
\partial_{t} V+\nabla \eta+\varepsilon & {\left[\frac{1}{4} \nabla\left(\eta^{2}\right)+\frac{3}{2}\left(\begin{array}{c}
\partial_{x}\left(V_{1}^{2}\right) \\
\partial_{y}\left(V_{2}^{2}\right)
\end{array}\right)+\frac{1}{4}\left(\begin{array}{c}
\partial_{x}\left(V_{2}^{2}\right) \\
\partial_{y}\left(V_{1}^{2}\right)
\end{array}\right)+\frac{1}{2}\left(\begin{array}{c}
\partial_{y}\left(V_{1} V_{2}\right) \\
\partial_{x}\left(V_{1} V_{2}\right)
\end{array}\right)\right] } \\
+ & \varepsilon\left(a \Delta \nabla \eta-b \Delta \partial_{t} V\right)=0 \\
\partial_{t} \eta+\nabla \cdot V+ & +\frac{\varepsilon}{2} \nabla \cdot(\eta V)+\varepsilon\left(c \Delta \nabla \cdot V-d \Delta \partial_{t} \eta\right)=0,
\end{aligned}
$$

where $V=\left(V_{1}, V_{2}\right)^{T}$ denotes the horizontal velocity field at height $\theta$ and in our present scaling it is naturally required that $0 \leq \theta \leq 1$. The constants $a, b$, $c$ and $d$ appearing in the equation are

$$
a=\left(\frac{\theta^{2}}{2}-\frac{1}{6}\right) \lambda, b=\left(\frac{\theta^{2}}{2}-\frac{1}{6}\right)(1-\lambda), c=\frac{\left(1-\theta^{2}\right)}{2} \mu, d=\frac{\left(1-\theta^{2}\right)}{2}(1-\mu),
$$

where $\lambda$ and $\mu$ are any two real parameters. If one chooses the parameters so that $a=c$ and $b \geq 0, d \geq 0$, then this system is symmetric and it is well posed in $W^{k, \infty}\left(0, T / \varepsilon ; H^{s-3 k}\left(\mathbb{R}^{3}\right)\right)$ for any $k$ and $s$ such that $s-3 k>2$. Moreover, one has the exact conservation law

$$
\partial_{t} \int_{\mathbb{R}^{2}} V^{2}+\eta^{2}+\varepsilon\left(b|\nabla V|^{2}+d|\nabla \eta|^{2}\right)=0
$$

(see Proposition 2.4). 
Fully symmetric models turns out to be a powerful mathematical tool. Indeed, we are able to prove that appropriately smooth solution of the full equations (1.1)-(1.4) can be approximated by solutions of these symmetric systems with an error which is at most of order $\varepsilon^{2} t$, uniformly for $t \in[0, T / \varepsilon]$. We then show that in two space dimensions, smooth solutions of any of the Bona-Chen-Saut systems differ from solutions of a symmetric system by at most a quantity of order $\varepsilon^{2} t$ on the same long time scale $\varepsilon^{-1}$. The latter results give a satisfactory rigorous foundation to the use of any of the well posed versions of these models to describe two-dimensional surface water waves in the long-wave or Boussinesq regime.

D. Lannes proved recently [16] that the water-waves equations are wellposed in finite depth ( $\mathrm{Wu}$ proved earlier the case of infinite depth [22]). However, applying this result to the present case of long-waves does not yield directly that the existence time is large, of order $O(1 / \varepsilon)$. Thus, our threedimensional results are couched in terms of solutions of the Euler equations that may exist, with the extra assertion that such solutions necessarily do persist on the relevant long time scale.

Our results apply both to the equations posed with the bottom comprising the entire space $\mathbb{R}^{d}, d=1$ or 2 with function-space restrictions that imply solutions decay to zero at infinity and to the periodic initial-value problems. This latter aspect of the theory will be seen to bear upon the prospect of using a decoupled type of approximation as exemplified by (1.6) in the two dimensional context.

Returning briefly to the issue of approximation via a decoupled system in the two dimensional situation, remark first that laboratory experiments and real world flows arising in geophysical contexts often show nonlinear coupling effects between counter-propagating waves. This apparent contradiction with the result of [19] quoted above appears to derive from two sources. First, in practice, the parameter $\varepsilon$ is not so small. (Values of $\varepsilon$ on the order of 0.3 find regular appearance in situations where approximations to the Euler equations are used to model real waves.) Secondly, the Schneider-Wayne result subsists upon an assumption of a definite rate of decrease of the relevant wave motion at infinity. Many wave regimes arising in the laboratory or in field situations are of a quasi-periodic nature and certainly do not fit the approximation of tending to zero at infinity at a substantial rate, at least on the spatial and temporal scales where the models might possibly be useful. Indeed, as will become apparent in the analysis presented in Section 5, a decoupled approximation such as (1.6) does not present the same convergence rate to associated solutions of the Euler equations (1.1)-(1.4) as does the coupled Boussinesq equations presented here and in [5] in the situation where the initial disturbance has only function class restrictions and not a definite rate of approach to zero at infinity. In the periodic case, the decoupled system fails to provide a useful approximation to the Euler equations, in contrast to the coupled systems developed here and in [5].

In the next subsection, a more detailed view of the theory developed herein 
is presented. The plan of the remainder of the paper is as follows. Section 2 is devoted to the derivation of the symmetric systems to which frequent reference has just been made. The exact relationship between the symmetric systems and the full Euler equations is investigated in Section 3, while in Section 4, it is proven in the two-dimensional case that the exact solutions of the Euler equations approximately satisfy the class of models $S$ put forward by Bona, Chen and Saut. A detailed analysis of the decoupled formulation is the subject of the last section.

\subsection{Description of the results}

There are three principal types of results in the technical elaboration of our theory, namely, consistency, existence and convergence results. To describe them, we need to discuss in a preliminary fashion the different systems involved.

The primary system on which everything developed here is based is the Euler system (1.1)-(1.4). From the Euler system, we obtain the classical Boussinesq system by making the long wave and small amplitude assumptions outlined above and then expanding appropriately in the small parameter $\varepsilon$, and dropping terms that are formally of order higher than linear in $\varepsilon$. Boussinesq's original system belongs to the wide class obtained by Bona, Chen and Saut [5] which is here denoted by $S$. All these systems have the same nonlinear structure as the original Boussinesq system; they differ one from the other only in their modeling of dispersion. As mentioned above, they are all formally equivalent. The second class of systems, denoted $S^{\prime}$, is a new one. It is obtained from $S$ by nonlinear changes of variables which renders symmetric the nonlinear hyperbolic portion (the non-dispersive part of the system). Systems of the class $S^{\prime}$ are also formally equivalent to the systems of $S$, and hence formally good approximations of the full Euler equations on the long time scale characterized by $\varepsilon^{-1}$. The final class marked for discussion here is denoted $\Sigma$ and is in fact the subclass of $S^{\prime}$ consisting of those systems for which both the dispersive and the nonlinear part are symmetric. The class $\Sigma$ is nonempty. Indeed, the sample system displayed in Section 1.1 is a member. Systems which belong to $\Sigma$ are conservative in a sense hinted at in Section 1.1, and which will be made precise presently.

In what follows, we will prove theorems of existence for systems in $\Sigma$ and of convergence of their solutions to those of the full Euler equations, including bounds on the rate of convergence. We will also prove comparison theorems between solutions of the systems in $\Sigma$ and solutions of the other systems under discussion.

A crucial ingredient in our analysis will be the notion of consistency which is presented now. Consider the general system

$$
\partial_{t} \mathcal{U}+A\left(\partial_{X}\right) \mathcal{U}+\varepsilon B(\mathcal{U})\left(\partial_{X} \mathcal{U}\right)+\varepsilon\left(C\left(\partial_{X}^{3} \mathcal{U}\right)+D\left(\partial_{X}^{2} \partial_{t} \mathcal{U}\right)\right)=0
$$

where $\mathcal{U}(t, X):[0, T / \varepsilon] \times \mathbb{R}^{d} \rightarrow \mathbb{R}^{p}, T$ is a fixed, positive constant, $p$ is an integer, $X=(x, y)$ if $d=2$ and $X=x$ if $d=1, A\left(\partial_{X}\right)=A_{1} \partial_{x}+A_{2} \partial_{y}$ 
with $A_{1}$ and $A_{2}$ constant matrices, $B(\mathcal{U})\left(\partial_{X} \mathcal{U}\right)=B_{1}(\mathcal{U}) \partial_{x} \mathcal{U}+B_{2}(\mathcal{U}) \partial_{y} \mathcal{U}$, $C\left(\partial_{X}^{3} \mathcal{U}\right)=\sum_{i, j, k=1}^{d} C_{i j k} \partial_{i j k}^{3} \mathcal{U}$ and $D\left(\partial_{X}^{2} \partial_{t} \mathcal{U}\right)=\sum_{i, j=1}^{d} D_{i j} \partial_{i j}^{2} \partial_{t} \mathcal{U}$. To be concrete, we can presume that $B_{1}$ and $B_{2}$ are polynomial in the components of $\mathcal{U}$ and that $C_{i j k}$ and $D_{i j}$ are constant matrices, though this is not necessary for some of what follows. All the systems in the classes $S, S^{\prime}$ and $\Sigma$, can be written in the form (1.7), so it suffices for the purposes at hand to provide a definition of consistency within the context of (1.7).

Definition 1.1 Let $s \geq 0, \sigma \geq s, \varepsilon_{0}>0$ and $T>0$ be given. A family $\left\{\mathcal{U}^{\varepsilon}\right\}_{0<\varepsilon<\varepsilon_{0}}$ which is bounded independently of $\varepsilon$ in $W^{1, \infty}\left(0, T / \varepsilon ; H^{\sigma}\left(\mathbb{R}^{d}\right)\right)$ is consistent with the system (1.7) if

$$
\partial_{t} \mathcal{U}^{\varepsilon}+A\left(\partial_{X}\right) \mathcal{U}^{\varepsilon}+\varepsilon B\left(\mathcal{U}^{\varepsilon}\right)\left(\partial_{X} \mathcal{U}^{\varepsilon}\right)+\varepsilon\left(C\left(\partial_{X}^{3} \mathcal{U}^{\varepsilon}\right)+D\left(\partial_{X}^{2} \partial_{t} \mathcal{U}^{\varepsilon}\right)\right)=\varepsilon^{2} \mathcal{R}^{\varepsilon}
$$

where the family $\left\{\mathcal{R}^{\varepsilon}\right\}_{0<\varepsilon<\varepsilon_{0}}$ is bounded in $L^{\infty}\left(0, T / \varepsilon ; H^{s}\left(\mathbb{R}^{d}\right)\right)$ for $0<\varepsilon<\varepsilon_{0}$. When the values of $\sigma$ and $s$ are important, we will say the family $\left\{\mathcal{U}^{\varepsilon}\right\}_{0<\varepsilon<\varepsilon_{0}}$ is consistent with regularity $\sigma$ and $s$.

One of the technical goals of this paper is to establish rigorous consistency results for systems of the classes $S, S^{\prime}$ and $\Sigma$ and to prove related existence and convergence results. Here is a more detailed account of what is in view.

i. Consistency results. Let $\left\{\left(\phi^{\varepsilon}, \eta^{\varepsilon}\right)\right\}$ be a family of solutions of the Euler equations (1.1)-(1.4) for some open interval of $\varepsilon$ of the form $\left(0, \varepsilon_{0}\right)$, say, where $\varepsilon_{0}>0$. Define $V^{\varepsilon}:=\nabla \psi^{\varepsilon}$ with $\psi^{\varepsilon}(t, X):=\phi\left(X, 1+\varepsilon \eta^{\varepsilon}(t, X)\right)$. If $\left\{\left(V^{\varepsilon}, \eta^{\varepsilon}\right)\right\}$ is bounded in $W^{1, \infty}\left(0, T / \varepsilon ; H^{\sigma}\left(\mathbb{R}^{d}\right)\right)$ for some $\sigma$ large enough, it is established in Theorem 4.2 that $\left\{\left(V^{\varepsilon}, \eta^{\varepsilon}\right)\right\}$ is consistent with the Boussinesq system. This result is in the general spirit of results of Craig, Schantz, Sulem and Sulem in [13] and [12]. A direct approach is mounted to prove this result which avoids use of the singular integral associated to the Dirichlet-toNeumann operator for the flow domain.

We also prove that any function consistent with any one of the systems of class $S$ is, up to a linear change of variables, consistent with any other system of $S$. The linear change of variables (taken from [5]) corresponds physically to taking as a new independent variable the horizontal velocity at a different height above the bottom (see Proposition 2.1). Similarly, it is shown that if $\left\{\left(V^{\varepsilon}, \eta^{\varepsilon}\right)\right\}$ is consistent with a system in $S$ then $\left\{\left(\widetilde{V^{\varepsilon}}, \widetilde{\eta^{\varepsilon}}\right)\right\}$ obtained from $\left\{\left(V^{\varepsilon}, \eta^{\varepsilon}\right)\right\}$ by the aforementioned nonlinear changes of variables is consistent with the systems of the class $S^{\prime}$ (which have symmetric nonlinear part, remember). A key presumption in the three-dimensional theory at this point is the irrotationality of the flow expressed as the vanishing of the curl of the velocity field everywhere in the flow domain (see Proposition 2.2). Moreover, we prove in Proposition 2.3 that any function consistent with a system in $S^{\prime}$ is also consistent with all the other members of $S^{\prime}$ (again, up to a linear change of variables). 
ii. Existence results. For the systems of class $S$, the local well posedness for the Cauchy problem has been discussed in some detail in [5] and [6]. Necessary and sufficient conditions for the well posedness of the associated linear problems was given in [5]. It was posited that the systems that are linearly ill posed are unlikely to be nonlinearly well posed and they were discarded from the discussion in [6]. In the latter reference, local well posedness was demonstrated for all the systems that were determined to be linearly well posed save for one highly degenerate case. However, when written in the scaling favoured here, the time of existence for most of these systems using the theory in [6] is only on the order of $\varepsilon^{-1 / 2}$. (However, certain of the systems in $S$ have a global existence theory owing to a special, Hamiltonian structure.) For the present purposes, it is convenient to have an existence theory on the time interval $[0, T / \varepsilon]$ where $T$ may depend upon the order one initial data, but is independent of $\varepsilon$, together with $\varepsilon$-independent bounds for the solutions in $W^{1, \infty}\left(0, T / \varepsilon ; H^{\sigma}\left(\mathbb{R}^{d}\right)\right)$ for $\sigma$ large enough. Fortunately, the systems of class $\Sigma$, which play a crucial role in our analysis anyway, are indeed locally well posed on the longer time scale of order $\varepsilon^{-1}$, as is shown in Proposition 2.4.

iii. Convergence results. The most fundamental of our convergence results, Theorem 3.1, concerns solutions of the systems within $\Sigma$. It states that corresponding to any family $\left\{\left(V^{\varepsilon}, \eta^{\varepsilon}\right)\right\}$ of functions consistent with one of the $\Sigma$-systems, there exists a family of exact solutions $\left\{\left(V_{\Sigma}^{\varepsilon}, \eta_{\Sigma}^{\varepsilon}\right)\right\}$ of the relevant system satisfying

$$
\left|\left(V^{\varepsilon}, \eta^{\varepsilon}\right)-\left(V_{\Sigma}^{\varepsilon}, \eta_{\Sigma}^{\varepsilon}\right)\right|_{L^{\infty}\left(0, t ; H^{s}\left(\mathbb{R}^{d}\right)\right)}=O\left(\varepsilon^{2} t\right)
$$

for all $t \in[0, T / \varepsilon]$. This error estimate is easily established owing to the symmetry of the systems in the class $\Sigma$. From this central result, one deduces at once that the asymptotic behavior of any family of functions $\left\{\left(V^{\varepsilon}, \eta^{\varepsilon}\right)\right\}$, consistent with one of the systems of class $S$ or $S^{\prime}$ can be described in terms of an exact solution $\left\{\left(V_{\Sigma}^{\varepsilon}, \eta_{\Sigma}^{\varepsilon}\right)\right\}$ to one of the symmetric systems of class $\Sigma$ (see our Corollaries 3.1 and 3.2).

The principal convergence result concerns the asymptotics of the full Euler equations $(1.1)-(1.4)$. It is demonstrated that for any family $\left\{\left(V^{\varepsilon}, \eta^{\varepsilon}\right)\right\}$ of solutions of the Euler equations and for any system in the class $\Sigma$, there exists a family $\left\{\left(V_{\Sigma}^{\varepsilon}, \eta_{\Sigma}^{\varepsilon}\right)\right\}$ of solutions to this $\Sigma$ - system with the following property. Let $\left\{\left(V_{\text {app }}^{\varepsilon}, \eta_{\text {app }}^{\varepsilon}\right)\right\}$ be the family of functions obtained by applying to $\left\{\left(V_{\Sigma}^{\varepsilon}, \eta_{\Sigma}^{\varepsilon}\right)\right\}$ an approximate inverse of the change of variables that arose in deriving the class $S^{\prime}$ from the class $S$, followed by the inverse of the transformation that came to the fore in deriving elements of the class $S$ from the classical Boussinesq equation. This results in a set of variables that are in principal directly comparable to the Euler variables. Using the preceding theory, one readily adduces that

$$
\left|\left(V^{\varepsilon}, \eta^{\varepsilon}\right)-\left(V_{a p p}^{\varepsilon}, \eta_{a p p}^{\varepsilon}\right)\right|_{L^{\infty}\left(0, t ; H^{s}\left(\mathbb{R}^{d}\right)\right)}=O\left(\varepsilon^{2} t\right)
$$

for all $t \in[0, T / \varepsilon]$ (see Theorems 3.2 and 3.3). 
Combining these convergence results yields the overall point in view here, which is that solutions of any of the approximate systems in view yield good approximations to the full Euler equations on the long time scale $\varepsilon^{-1}$ where nonlinear and dispersive effects can have an order one relative effect on the velocity field and the wave profile. In particular, these results leave one free to choose an approximate system with good mathematical properties for the modelling task at hand. This freedom can be very helpful when questions of the design of numerical schemes or the imposition of non-homogeneous boundary conditions arise. In many cases, the dispersive perturbation of a symmetric hyperbolic system, the hallmark of the systems in the class $\Sigma$, appears to be very convenient, for example.

A couple of further points are worth emphasis. Note that for the two dimensional case, for any initial data $\left(v_{0}, \eta_{0}\right) \in H^{s}(\mathbb{R})^{2}$ for suitably large $s$, there exists a solution to the Euler equations on the relevant time scale. Thus in the two dimensional case, the asymptotic analysis is complete on the time scale $\varepsilon^{-1}$. In the three dimensional situation, our theory applies to solutions of the full Euler equations should they exist. Finally, we point again to Section 5 where we analyse the approximating power of uncoupled systems as in (1.6). The approximation estimates mentioned above are superior to those that obtain for a pair of uncoupled $\mathrm{KdV}$-equations in the absence of specific decay presumptions about the solutions. Moreover, the present theory remains valid in the periodic case, which is not the case for decoupled models.

Acknowledgment. Part of this work was done while T. Colin and D. Lannes were visiting J. Bona at the University of Illinois at Chicago; they want to thank UIC for its hospitality. This work was partially supported by the ACI Jeunes chercheurs du ministère de la Recherche "solutions oscillantes d'EDP", the GDR 2103 EAPQ CNRS, by the European network HYKE, funded by the EC as contract HPRN-CT-2002-00282, and by the United States National Science Foundation.

\section{Formal derivation of symmetric systems.}

\subsection{The class $S$ of Bona-Chen-Saut systems.}

The aim of this section is to recall the derivation of a class of model systems which, in the two dimensional setting, were put forward recently by Bona, Chen and Saut in [5] and [6]. We take as our starting point one of the original versions of the Boussinesq system, namely

$$
\begin{aligned}
& \partial_{t} V+\nabla \eta+\frac{\varepsilon}{2} \nabla|V|^{2}=O\left(\varepsilon^{2}\right), \\
& \partial_{t} \eta+\nabla \cdot V+\varepsilon\left(\nabla \cdot(\eta V)+\frac{1}{3} \Delta \nabla \cdot V\right)=O\left(\varepsilon^{2}\right),
\end{aligned}
$$


as $\varepsilon \rightarrow 0$, where $V$ connotes the horizontal velocity at the free surface and $\eta$ the deviation of the free surface from its rest position as before. Henceforth, the notation $\left(V_{0}, \eta_{0}\right)$ is reserved for the value of $(V, \eta)$ at $t=0$, which is to say, the initial data. As above, $\varepsilon$ is the small parameter measuring the amplitude to depth ratio and, on account of the assumption that the Stokes number is exactly equal to 1 , the square of the ratio of the depth to a typical wavelength. We give below in proposition 2.1 a precise sense of what is meant by the formal notation $O\left(\varepsilon^{2}\right)$ on the right-hand side of $(2.1)$.

Elementary potential theory shows that the horizontal velocity of the water at height $\theta$ (recall that in the present scaling, $\theta=1$ at the free surface and $\theta=0$ at the bottom) is approximately given by $V_{\theta}$, where

$$
V_{\theta}=\left(1-\frac{\varepsilon}{2}\left(1-\theta^{2}\right) \Delta\right)^{-1} V
$$

or equivalently

$$
V=\left(1-\frac{\varepsilon}{2}\left(1-\theta^{2}\right) \Delta\right) V_{\theta}
$$

Substituting the relation (2.2) into (2.1) leads to the system

$$
\begin{aligned}
& \partial_{t} V_{\theta}+\nabla \eta+\frac{\varepsilon}{2}\left(\nabla\left|V_{\theta}\right|^{2}-\left(1-\theta^{2}\right) \Delta \partial_{t} V_{\theta}\right)=O\left(\varepsilon^{2}\right) \\
& \partial_{t} \eta+\nabla \cdot V_{\theta}+\varepsilon\left(\nabla \cdot\left(\eta V_{\theta}\right)+\left(\frac{\theta^{2}}{2}-\frac{1}{6}\right) \Delta \nabla \cdot V_{\theta}\right)=O\left(\varepsilon^{2}\right)
\end{aligned}
$$

(cf. [5] in the two dimensional case). Note that the initial data for $V_{\theta}$ is

$$
\left(1-\frac{\varepsilon}{2}\left(1-\theta^{2}\right) \Delta\right)^{-1} V_{0}:=V_{\theta, 0}
$$

To introduce the BBM-version of these systems, remark that at the lowest formal order,

$$
\begin{aligned}
& \partial_{t} V_{\theta}=-\nabla \eta+O(\varepsilon), \\
& \partial_{t} \eta=-\nabla \cdot V_{\theta}+O(\varepsilon) .
\end{aligned}
$$

In consequence, the dispersive terms in (2.3) may be rewritten in the form

$$
\begin{gathered}
\Delta \partial_{t} V_{\theta}=(1-\mu) \Delta \partial_{t} V_{\theta}-\mu \Delta \nabla \eta+O(\varepsilon), \\
\Delta \nabla V_{\theta}=\lambda \Delta \nabla V_{\theta}-(1-\lambda) \Delta \partial_{t} \eta+O(\varepsilon),
\end{gathered}
$$

without loss of formal accuracy in terms of powers of $\varepsilon$, where $\lambda$ and $\mu$ are two arbitrary parameters. Using (2.4) in (2.3) gives the system

$$
\left\{\begin{array}{l}
\partial_{t} V_{\theta}+\nabla \eta+\varepsilon\left(\frac{1}{2} \nabla\left|V_{\theta}\right|^{2}-(1-\mu) \frac{\left(1-\theta^{2}\right)}{2} \Delta \partial_{t} V_{\theta}+\mu \frac{\left(1-\theta^{2}\right)}{2} \Delta \nabla \eta\right)=O\left(\varepsilon^{2}\right), \\
\partial_{t} \eta+\nabla \cdot V_{\theta}+\varepsilon\left(\nabla \cdot\left(\eta V_{\theta}\right)+\left(\frac{\theta^{2}}{2}-\frac{1}{6}\right) \lambda \Delta \nabla \cdot V_{\theta}-\left(\frac{\theta^{2}}{2}-\frac{1}{6}\right)(1-\lambda) \Delta \partial_{t} \eta\right)=O\left(\varepsilon^{2}\right) .
\end{array}\right.
$$


The class $S$ is just all the systems of the form $S_{\theta, \lambda, \mu}$ where $0 \leq \theta \leq 1$ and $\mu$ and $\lambda$ are arbitary real numbers. These are written in the compact form

$$
S_{\theta, \lambda, \mu}\left\{\begin{array}{l}
\partial_{t} V+\nabla \eta+\varepsilon\left(\frac{1}{2} \nabla|V|^{2}+a \Delta \nabla \eta-b \Delta \partial_{t} V\right)=0, \\
\partial_{t} \eta+\nabla \cdot V+\varepsilon\left(\nabla \cdot(\eta V)+c \Delta \nabla \cdot V-d \Delta \partial_{t} \eta\right)=0 .
\end{array}\right.
$$

with

$$
\begin{aligned}
& a=\frac{\left(1-\theta^{2}\right)}{2} \mu, \quad b=\frac{\left(1-\theta^{2}\right)}{2}(1-\mu) \\
& c=\left(\frac{\theta^{2}}{2}-\frac{1}{6}\right) \lambda, \quad d=\left(\frac{\theta^{2}}{2}-\frac{1}{6}\right)(1-\lambda)
\end{aligned}
$$

as in [5] in the two dimensional case.

Our first consistency result (in the sense of Definition 1.1) is provided in the following proposition.

Proposition 2.1 Let $\lambda$ and $\mu$ be given and suppose $\theta, \theta_{1} \in[0,1]$. Let $\left(V^{\varepsilon}, \eta^{\varepsilon}\right)$ be consistent with the system $S_{\theta, \lambda, \mu}$ and let $V_{1}^{\varepsilon}$ be defined by

$$
V_{1}^{\varepsilon}=\left(1-\frac{\varepsilon}{2}\left(1-\theta_{1}^{2}\right) \Delta\right)^{-1}\left(1-\frac{\varepsilon}{2}\left(1-\theta^{2}\right) \Delta\right) V^{\varepsilon}
$$

Then $\left(V_{1}^{\varepsilon}, \eta^{\varepsilon}\right)$ is consistent with $S_{\theta_{1}, \lambda, \mu}$. Moreover, for all $\left(\lambda_{1}, \mu_{1}\right) \in \mathbb{R}^{2}$, $\left(V_{1}^{\varepsilon}, \eta^{\varepsilon}\right)$ is consistent with $S_{\theta_{1}, \lambda_{1}, \mu_{1}}$.

\section{Proof.}

This is clear thanks to the relation (2.2).

As discussed already, for a long wave model for the water-wave problem to be useful, it must have a local existence theory for order one initial data that provides smooth solutions at least on time intervals of the form $[0, T / \varepsilon]$. Moreover, solutions with fixed initial data in the variables in force here must be bounded with respect to $\varepsilon$ in $W^{1, \infty}\left(0, T / \varepsilon ; H^{s}\left(\mathbb{R}^{d}\right)\right)$ for suitably large values of $s$. For most of the members of the class $S$, even those which are locally well posed, there is no theory of this nature. (There is a sparse subclass of the systems in $S$ that have a global existence theory and these of course conform to the time-scale requirements. However, even in these cases, there is no analysis showing the solutions are bounded independently of small values of $\varepsilon$ ).

This situation will be rectified by making use of the symmetric systems which are the subject of the next subsection. 


\subsection{The class $S^{\prime}$ of nonlinearly symmetric systems}

In the previous section, we recalled how to obtain formally equivalent model systems from the Boussinesq system by making changes of variables that had the effect of modifying the dispersive part of the model. In this section, attention is given to the nonlinear part. The formal zero-dispersion limit of the Boussinesq system (2.1) is the system

$$
\left\{\begin{array}{l}
\partial_{t} V+\nabla \eta+\frac{\varepsilon}{2} \nabla|V|^{2}=0 \\
\partial_{t} \eta+\nabla \cdot V+\varepsilon \nabla \cdot(\eta V)=0
\end{array}\right.
$$

of hyperbolic conservation laws. It is handy to write (2.6) in the form

$$
\partial_{t}\left(\begin{array}{c}
V \\
\eta
\end{array}\right)+A_{1}(V, \eta) \partial_{x}\left(\begin{array}{c}
V \\
\eta
\end{array}\right)+A_{2}(V, \eta) \partial_{y}\left(\begin{array}{c}
V \\
\eta
\end{array}\right)=0
$$

where

$$
A_{1}(V, \eta)=\left(\begin{array}{lll}
\varepsilon V_{1} & \varepsilon V_{2} & 1 \\
0 & 0 & 0 \\
1+\varepsilon \eta & 0 & \varepsilon V_{1}
\end{array}\right)
$$

and

$$
A_{2}(V, \eta)=\left(\begin{array}{clc}
0 & 0 & 0 \\
\varepsilon V_{1} & \varepsilon V_{2} & 1 \\
0 & 1+\varepsilon \eta & \varepsilon V_{2}
\end{array}\right),
$$

in the three dimensional case. In two space dimensions, this becomes

$$
\partial_{t}\left(\begin{array}{l}
v \\
\eta
\end{array}\right)+A(v, \eta) \partial_{x}\left(\begin{array}{l}
v \\
\eta
\end{array}\right)=0
$$

where

$$
A(v, \eta)=\left(\begin{array}{cc}
\varepsilon v & 1 \\
1+\varepsilon \eta & \varepsilon v
\end{array}\right) .
$$

Obviously, whatever the space dimension is, these systems of conservation laws are not symmetric. However, a symmetrizer in the fully three dimensional case is

$$
\left(\begin{array}{lll}
1+\varepsilon \eta & 0 & \varepsilon V_{1} \\
0 & 1+\varepsilon \eta & \varepsilon V_{2} \\
\varepsilon V_{1} & \varepsilon V_{2} & 1
\end{array}\right)
$$


Note that in two space dimensions, the symmetrizer specializes to

$$
\left(\begin{array}{cc}
1+\varepsilon \eta & \varepsilon v \\
\varepsilon v & 1
\end{array}\right)
$$

but that

$$
\left(\begin{array}{ll}
1+\varepsilon \eta & 0 \\
0 & 1
\end{array}\right)
$$

can also be used.

Independently of the dimension, these symmetrizers are not compatible with the dispersion terms. Therefore, they cannot be used for adducing solutions to the Cauchy problem. This disappointing observation leads one to search for another strategy for obtaining "equivalent" systems, in the sense of the preceding subsection, that are symmetric as regards their nonlinear portion. Consider the nonlinear change of variables

$$
\tilde{V}=V\left(1+\frac{\varepsilon}{2} \eta\right)
$$

and compute the equations satisfied by $\tilde{V}$ and $\eta$ :

$$
\begin{gathered}
\partial_{t} \tilde{V}=\partial_{t} V\left(1+\frac{\varepsilon}{2} \eta\right)+\tilde{V} \frac{\varepsilon}{2} \partial_{t} \eta+O\left(\varepsilon^{2}\right), \\
=-\left[\nabla \eta+\frac{\varepsilon}{2} \nabla|V|^{2}\right]\left(1+\frac{\varepsilon}{2} \eta\right)-\frac{\varepsilon}{2} \tilde{V} \nabla \cdot \tilde{V}+O\left(\varepsilon^{2}\right), \\
=-\nabla \eta-\varepsilon\left(\frac{1}{2} \nabla|\tilde{V}|^{2}+\frac{1}{4} \nabla|\eta|^{2}+\frac{1}{2} \tilde{V} \nabla \cdot \tilde{V}\right)+O\left(\varepsilon^{2}\right) .
\end{gathered}
$$

It follows

$$
\partial_{t} \tilde{V}+\nabla \eta+\varepsilon\left(\frac{1}{2} \nabla|\tilde{V}|^{2}+\frac{1}{4} \nabla|\eta|^{2}+\frac{1}{2} \tilde{V} \nabla \cdot \tilde{V}\right)=O\left(\varepsilon^{2}\right) .
$$

On the other hand:

$$
\begin{gathered}
\partial_{t} \eta=-\nabla \cdot V-\varepsilon \nabla \cdot(\eta V)+O\left(\varepsilon^{2}\right), \\
=-\nabla \cdot\left[\tilde{V}\left(1-\frac{\varepsilon}{2} \eta\right)\right]-\varepsilon \nabla \cdot(\eta V)+O\left(\varepsilon^{2}\right), \\
=-\nabla \cdot \tilde{V}-\varepsilon\left(\nabla \cdot(\eta \tilde{V})-\frac{1}{2} \nabla(\eta \tilde{V})\right)+O\left(\varepsilon^{2}\right) .
\end{gathered}
$$

Therefore

$$
\partial_{t} \eta+\nabla \cdot \tilde{V}+\frac{\varepsilon}{2} \nabla \cdot(\eta \tilde{V})=O\left(\varepsilon^{2}\right)
$$

The system formed by (2.8) and (2.9) is symmetric in $1-D$ but not in $2-D$. Indeed, it can be written (omitting the tilde):

$$
\partial_{t} V_{1}+\partial_{x} \eta+\varepsilon\left(V_{1} \partial_{x} V_{1}+V_{2} \partial_{x} V_{2}+\frac{1}{2} \eta \partial_{x} \eta+\frac{1}{2} V_{1}\left(\partial_{x} V_{1}+\partial_{y} V_{2}\right)\right)=O\left(\varepsilon^{2}\right),
$$




$$
\begin{gathered}
\partial_{t} V_{2}+\partial_{y} \eta+\varepsilon\left(V_{1} \partial_{y} V_{1}+V_{2} \partial_{y} V_{2}+\frac{1}{2} \eta \partial_{y} \eta+\frac{1}{2} V_{2}\left(\partial_{x} V_{1}+\partial_{y} V_{2}\right)\right)=O\left(\varepsilon^{2}\right), \\
\partial_{t} \eta+\partial_{x} V_{1}+\partial_{y} V_{2}+\frac{\varepsilon}{2}\left(\partial_{x} \eta V_{1}+\eta \partial_{x} V_{1}+\partial_{y} \eta V_{2}+\eta \partial_{y} V_{2}\right)=O\left(\varepsilon^{2}\right),
\end{gathered}
$$

or equivalently:

$$
\begin{aligned}
& \partial_{t}\left(\begin{array}{c}
V_{1} \\
V_{2} \\
\eta
\end{array}\right)+\left(\begin{array}{ccc}
\frac{3 \varepsilon}{2} V_{1} & \varepsilon V_{2} & 1+\frac{\varepsilon}{2} \eta \\
\frac{\varepsilon}{2} V_{2} & 0 & 0 \\
1+\frac{\varepsilon}{2} \eta & 0 & \frac{\varepsilon}{2} V_{1}
\end{array}\right) \partial_{x}\left(\begin{array}{c}
V_{1} \\
V_{2} \\
\eta
\end{array}\right) \\
& +\left(\begin{array}{ccc}
0 & \frac{\varepsilon}{2} V_{1} & 0 \\
\varepsilon V_{1} & \frac{3 \varepsilon}{2} V_{2} & 1+\frac{\varepsilon}{2} \eta \\
0 & 1+\frac{\varepsilon}{2} \eta & \frac{\varepsilon}{2} V_{2}
\end{array}\right) \partial_{y}\left(\begin{array}{l}
V_{1} \\
V_{2} \\
\eta
\end{array}\right)=O\left(\varepsilon^{2}\right) .
\end{aligned}
$$

At this step, we use the 0 -curl condition. Indeed, when curl $\tilde{V}=O(\varepsilon)$, one can replace $\frac{\varepsilon}{2} V_{2} \partial_{x} V_{2}$ by $\frac{\varepsilon}{2} V_{2} \partial_{y} V_{1}$ in the first equation of (2.10) and $\frac{\varepsilon}{2} V_{1} \partial_{y} V_{1}$ by $\frac{\varepsilon}{2} V_{1} \partial_{x} V_{2}$ in the second one. The system becomes:

$$
\left\{\begin{array}{l}
\partial_{t} V_{1}+\partial_{x} \eta+\varepsilon\left(\frac{1}{4} \partial_{x}\left(\eta^{2}\right)+\frac{3}{2} \partial_{x}\left(V_{1}^{2}\right)+\frac{1}{4} \partial_{x}\left(V_{2}^{2}\right)+\frac{1}{2} \partial_{y}\left(V_{1} V_{2}\right)\right)=O\left(\varepsilon^{2}\right), \\
\partial_{t} V_{2}+\partial_{y} \eta+\varepsilon\left(\frac{1}{4} \partial_{y}\left(\eta^{2}\right)+\frac{1}{4} \partial_{y}\left(V_{1}^{2}\right)+\frac{3}{2} \partial_{y}\left(V_{2}^{2}\right)+\frac{1}{2} \partial_{x}\left(V_{1} V_{2}\right)\right)=O\left(\varepsilon^{2}\right), \\
\partial_{t} \eta+\partial_{x} V_{1}+\partial_{y} V_{2}+\frac{\varepsilon}{2}\left(\partial_{x}\left(\eta V_{1}\right)+\partial_{y}\left(\eta V_{2}\right)\right)=O\left(\varepsilon^{2}\right) .
\end{array}\right.
$$

We now introduce the new class $S^{\prime}$ of systems $S_{\theta, \lambda, \mu}^{\prime}$ :

$$
S_{\theta, \lambda, \mu}^{\prime}\left\{\begin{array}{l}
\partial_{t} V+\nabla \eta+\varepsilon\left(\frac{1}{4} \nabla \eta^{2}+\frac{3}{2}\left(\begin{array}{c}
\partial_{x}\left(V_{1}^{2}\right) \\
\partial_{y}\left(V_{2}^{2}\right)
\end{array}\right)\right. \\
\left.+\frac{1}{4}\left(\begin{array}{c}
\partial_{x}\left(V_{2}^{2}\right) \\
\partial_{y}\left(V_{1}^{2}\right)
\end{array}\right)+\frac{1}{2}\left(\begin{array}{c}
\partial_{y}\left(V_{1} V_{2}\right) \\
\partial_{x}\left(V_{1} V_{2}\right)
\end{array}\right)+a \Delta \nabla \eta-b \Delta \partial_{t} V\right)=0 \\
\partial_{t} \eta+\nabla \cdot V+\varepsilon\left(\frac{1}{2} \nabla(\eta V)+c \Delta \nabla \cdot V-d \Delta \partial_{t} \eta\right)=0
\end{array}\right.
$$


where $a, b, c, d$ are given by (2.5). The previous computations allow us to write the folllowing propostion.

Proposition 2.2 If $\left(V^{\varepsilon}, \eta^{\varepsilon}\right)$ is consistent with $S_{\theta, \lambda, \mu}$ and nearly irrotationnal in the sense that curl $V^{\varepsilon}=O(\varepsilon)$, then $\left(\tilde{V}^{\varepsilon}, \eta^{\varepsilon}\right)$ defined by $\tilde{V}^{\varepsilon}=V^{\varepsilon}\left(1+\frac{\varepsilon}{2} \eta^{\varepsilon}\right)$ is consistent with $S_{\theta, \lambda, \mu}^{\prime}$.

It is also clear that:

Proposition 2.3 Proposition 2.1 remains true if one takes systems of $S^{\prime}$ instead of $S$.

\subsection{The symmetric class.}

We consider the subclass of $S^{\prime}$ for which $a=c, b \geq 0$ and $d \geq 0$. We denote this (non-empty!) class by $\Sigma$.

Proposition 2.4 Let us fix $\theta, \lambda, \mu$ such that system (2.12) is in the class $\Sigma$. For all $s>\frac{d}{2}+1$, for all $\left(V_{0}, \eta_{0}\right) \in\left(H^{s}\left(\mathbb{R}^{d}\right)\right)^{d+1}$, there exist $T_{0}>0$ independent of $\varepsilon$ and a unique $(V, \eta) \in \mathcal{C}\left(\left[0, \frac{T_{0}}{\varepsilon}\right] ;\left(H^{s}\left(\mathbb{R}^{d}\right)\right)^{d+1}\right)$ solution to (2.12) such that $(V, \eta)(t=0)=\left(V_{0}, \eta_{0}\right)$.

Moreover, there exists $C_{0}>0$ independent of $\varepsilon$ such that

$$
|(V, \eta)|_{W^{k, \infty}\left(0, \frac{T_{0}}{\varepsilon} ; H^{s-3 k}\right)} \leq C_{0}
$$

for all $k$ such that $s-3 k>d / 2+1$.

One also has the following conservation law:

$$
\partial_{t} \int V^{2}+\eta^{2}+\varepsilon b|\nabla V|^{2}+d \varepsilon|\nabla \eta|^{2}=0
$$

\section{Proof.}

It is done using standard energy estimates for hyperbolic symmetric quasilinear systems. Note that the dispersive part does not interact with these estimates because of the relationships $a=c, b \geq 0$ and $d \geq 0$. The conservation of the energy follows from the following straightforward computation: one multiplies the system by $\left(\begin{array}{c}V \\ \eta\end{array}\right)$ and integrates. One gets for the nonlinear part:

$$
\begin{gathered}
\int \frac{1}{2} \eta \partial_{x} \eta V_{1}+3 V_{1}^{2} \partial_{x} V_{1}+\frac{1}{2} V_{2} \partial_{x} V_{2} V_{1}+\frac{1}{2} \partial_{y} V_{1} V_{2} V_{1} \\
+\frac{1}{2} V_{1} \partial_{y} V_{2} V_{1}+\frac{1}{2} \eta \partial_{y} \eta V_{2}+\frac{1}{2} V_{1} \partial_{y} V_{1} V_{2}+3 V_{2} \partial_{y} V_{2} V_{2} \\
+\frac{1}{2} \partial_{x} V_{1} V_{2}^{2}+\frac{1}{2} V_{1} \partial_{x} V_{2} V_{2}+\frac{1}{2} \eta^{2} \partial_{x} V_{1}+\frac{1}{2} \partial_{x} \eta V_{1} \eta+\frac{1}{2} \eta^{2} \partial_{y} V_{2}+\frac{1}{2} \partial_{y} \eta V_{2} \eta
\end{gathered}
$$




$$
\begin{gathered}
=\int \eta \partial_{x} \eta V_{1}+\frac{1}{2} \eta^{2} \partial_{x} V_{1}+\eta \partial_{y} \eta V_{2}+\frac{1}{2} \eta^{2} \partial_{y} V_{2} \\
+V_{2} \partial_{x} V_{2} V_{1}+\frac{1}{2} \partial_{x} V_{1} V_{2}^{2}+\partial_{y} V_{1} V_{2} V_{1}+\frac{1}{2} V_{1} \partial_{y} V_{2} V_{1}, \\
=\frac{1}{2} \int \partial_{x}\left(\eta^{2}\right) V_{1}+\eta^{2} \partial_{x} V_{1}+\partial_{y}\left(\eta^{2}\right) V_{2}+\eta^{2} \partial_{y} V_{2} \\
+\partial_{x}\left(V_{2}^{2}\right) V_{1}+\partial_{x} V_{1} V_{2}^{2}+\partial_{y}\left(V_{1}^{2}\right) V_{2}+V_{1}^{2} \partial_{y} V_{2}=0 .
\end{gathered}
$$

This ends the proof of the proposition.

\section{Error estimates.}

\subsection{The symmetric systems.}

In this section, we prove the fundamental estimates that state that any solution consistent with the symmetric system is near a solution of that system on time intervals of size $O\left(\frac{1}{\varepsilon}\right)$. We take $\theta, \lambda, \mu$ such that the system $S_{\theta, \lambda, \mu}^{\prime}$ given by (2.12) is in $\Sigma$, that is has symmetric dispersive and nonlinear parts. Such a system can be written:

$$
\begin{gathered}
\partial_{t} V+\nabla \eta+\varepsilon\left(\frac{1}{4} \nabla \eta^{2}+\frac{3}{2}\left(\begin{array}{c}
\partial_{x}\left(V_{1}^{2}\right) \\
\partial_{y}\left(V_{2}^{2}\right)
\end{array}\right)\right. \\
\left.+\frac{1}{4}\left(\begin{array}{c}
\partial_{x}\left(V_{2}^{2}\right) \\
\partial_{y}\left(V_{1}^{2}\right)
\end{array}\right)+\frac{1}{2}\left(\begin{array}{c}
\partial_{y}\left(V_{1} V_{2}\right) \\
\partial_{x}\left(V_{1} V_{2}\right)
\end{array}\right)+a \Delta \nabla \eta-b \Delta \partial_{t} V\right)=0, \\
\partial_{t} \eta+\nabla \cdot V+\varepsilon\left(\frac{1}{2} \nabla(\eta V)+a \Delta \nabla \cdot V-d \Delta \partial_{t} \eta\right)=0,
\end{gathered}
$$

with

$$
\begin{array}{lll}
a=\frac{\left(1-\theta^{2}\right)}{2} \mu, & b=\frac{\left(1-\theta^{2}\right)}{2}(1-\mu), & d=\left(\frac{\theta^{2}}{2}-\frac{1}{6}\right)(1-\lambda) \\
\frac{\left(1-\theta^{2}\right)}{2} \mu=\left(\frac{\theta^{2}}{2}-\frac{1}{6}\right) \lambda, & b \geq 0, & d \geq 0 .
\end{array}
$$

The result reads:

Theorem 3.1 Let us fix $\lambda, \mu, \theta$ satisfying (3.3) and $s \geq 0$. Let $\left(V^{\varepsilon}, \eta^{\varepsilon}\right)$ a family of functions bounded with respect to $\varepsilon$ in $W^{1, \infty}\left(0, \frac{T}{\varepsilon} ; H^{s+3}\left(\mathbb{R}^{d}\right)\right)$ for some $T>0$. If this family is consistent with (3.1)-(3.2) in the sense of Def. 1.1, then there exists $\left(V_{\Sigma}^{\varepsilon}, \eta_{\Sigma}^{\varepsilon}\right)$ an exact solution to (3.1)-(3.2) defined on $\left[0, \frac{T}{\varepsilon}\right]$ and such that

$$
\left|V^{\varepsilon}-V_{\Sigma}^{\varepsilon}\right|_{L^{\infty}\left(0, t ; H^{s}\left(\mathbb{R}^{d}\right)\right)}+\left|\eta^{\varepsilon}-\eta_{\Sigma}^{\varepsilon}\right|_{L^{\infty}\left(0, t ; H^{s}\left(\mathbb{R}^{d}\right)\right)} \leq C \varepsilon^{2} t \text { for all } t \in\left[0, \frac{T}{\varepsilon}\right] .
$$




\section{Proof.}

We write system (3.1)-(3.2) as a dispersive perturbation of an hyperbolic quasilinear symmetric system:

$$
\begin{aligned}
& \mathcal{L}\left(\begin{array}{c}
V \\
\eta
\end{array}\right):=\partial_{t}\left(\begin{array}{l}
V \\
\eta
\end{array}\right)+\left(\begin{array}{c}
\nabla \eta \\
\nabla \cdot V
\end{array}\right)+\varepsilon\left(A(V, \eta) \partial_{x}\left(\begin{array}{l}
V \\
\eta
\end{array}\right)\right. \\
& \left.+B(V, \eta) \partial_{y}\left(\begin{array}{c}
V \\
\eta
\end{array}\right)+a\left(\begin{array}{c}
\Delta \nabla \eta \\
\Delta \nabla \cdot V
\end{array}\right)-\Delta \partial_{t}\left(\begin{array}{c}
b V \\
d \eta
\end{array}\right)\right)=0 .
\end{aligned}
$$

The assumption of consistency made on $\left(V^{\varepsilon}, \eta^{\varepsilon}\right)$ means that

$$
\mathcal{L}\left(\begin{array}{c}
V^{\varepsilon} \\
\eta^{\varepsilon}
\end{array}\right)=\varepsilon^{2}\left(\begin{array}{c}
r_{1}^{\varepsilon} \\
r_{2}^{\varepsilon}
\end{array}\right)
$$

with $\left(r_{1}^{\varepsilon}, r_{2}^{\varepsilon}\right)$ bounded in $L^{\infty}\left(0, \frac{T}{\varepsilon} ; H^{s}\left(\mathbb{R}^{d}\right)\right)$. Now let $\left(V_{\Sigma}^{\varepsilon}, \eta_{\Sigma}^{\varepsilon}\right)$ be the solution of (3.4) such that $\left(V_{\Sigma}^{\varepsilon}, \eta_{\Sigma}^{\varepsilon}\right)(t=0)=\left(V^{\varepsilon}, \eta^{\varepsilon}\right)(t=0)$. This solution is defined at least on $\left[0, \frac{T_{0}}{\varepsilon}\right]$ with $T_{0}>0$ by proposition 2.4 . We write the system satisfied by the difference $\left(V^{\varepsilon}-V_{\Sigma}^{\varepsilon}, \eta^{\varepsilon}-\eta_{\Sigma}^{\varepsilon}\right)$ and performing standard energy estimates on it leads to the error given in theorem 3.1 on $\left[0, \frac{T_{1}}{\varepsilon}\right]$ where $T_{1}=\operatorname{Min}\left(T_{0}, T\right)$. By usual arguments, one can take $T_{1}=T$.

\subsection{Corollaries.}

The first corollary shows that the asymptotic behavior of any family $\left(V^{\varepsilon}, \eta^{\varepsilon}\right)$ consistent with a system of $S^{\prime}$ can be described in terms of the solution of one of the systems of $\Sigma$, via a pseudo-differential change of variables.

Corollary 3.1 Let $s \geq 0$ and $(\underline{\theta}, \underline{\lambda}, \mu)$ fixed satisfying (3.3). Assume that there exist a set of parameters $(\theta, \lambda, \mu)$ and a smooth enough family $\left(V^{\varepsilon}, \eta^{\varepsilon}\right)$ consistent with the system $S_{\theta, \lambda, \mu}^{\prime} \in S^{\prime}$ and defined on $\left[0, \frac{T}{\varepsilon}\right]$ for some $T>0$. Then the system $S_{\underline{\theta}, \lambda, \mu}^{\prime} \in \Sigma$ admits a unique family of solutions $\left(V_{\Sigma}^{\varepsilon}, \eta_{\Sigma}^{\varepsilon}\right)$ defined on $\left[0, \frac{T}{\varepsilon}\right]$ and with initial conditions $\left(V_{\Sigma, 0}^{\varepsilon}, \eta_{\Sigma, 0}^{\varepsilon}\right)$ defined as

$V_{\Sigma, 0}^{\varepsilon}=\left(1-\frac{\varepsilon}{2}\left(1-\underline{\theta}^{2}\right) \Delta\right)^{-1}\left(1-\frac{\varepsilon}{2}\left(1-\theta^{2}\right) \Delta\right) V^{\varepsilon}(t=0), \quad \eta_{\Sigma, 0}^{\varepsilon}=\eta^{\varepsilon}(t=0)$.

Moreover, one has the error estimate

$$
\begin{aligned}
\mid V^{\varepsilon}-\left(1-\frac{\varepsilon}{2}\left(1-\theta^{2}\right) \Delta\right)^{-1}(1 & \left.-\frac{\varepsilon}{2}\left(1-\underline{\theta}^{2}\right) \Delta\right)\left.V_{\Sigma}^{\varepsilon}\right|_{L^{\infty}\left(0, t ; H^{s}\left(\mathbb{R}^{d}\right)\right)} \\
& +\left|\eta^{\varepsilon}-\eta_{\Sigma}^{\varepsilon}\right|_{L^{\infty}\left(0, t ; H^{s}\left(\mathbb{R}^{d}\right)\right)} \leq C \varepsilon^{2} t,
\end{aligned}
$$

for all $t \in\left[0, \frac{T}{\varepsilon}\right]$. 


\section{Proof.}

From Prop. 2.3, it follows that

$$
\left(\widetilde{V}^{\varepsilon}, \widetilde{\eta}^{\varepsilon}\right):=\left(\left(1-\frac{\varepsilon}{2}\left(1-\underline{\theta}^{2}\right) \Delta\right)^{-1}\left(1-\frac{\varepsilon}{2}\left(1-\theta^{2}\right) \Delta\right) V^{\varepsilon}, \eta^{\varepsilon}\right)
$$

is consistent with $S_{\underline{\theta}, \underline{\lambda}, \underline{\mu}}^{\prime} \in \Sigma$.

From Prop. 2.4 one can deduce the existence of $\left(V_{\Sigma}^{\varepsilon}, \eta_{\Sigma}^{\varepsilon}\right)$ and from Th. 3.1, the error estimate between $\left(\widetilde{V}^{\varepsilon}, \widetilde{\eta}^{\varepsilon}\right)$ and $\left(V_{\Sigma}^{\varepsilon}, \eta_{\Sigma}^{\varepsilon}\right)$. Inverting the pseudo-differential change of variables then yields the result.

The same kind of property holds for systems of the original class $S$, but one must also perform a nonlinear change of variables.

Corollary 3.2 Let $s \geq 0$ and $(\underline{\theta}, \underline{\lambda}, \mu)$ fixed satisfying (3.3). Assume that there exist a set of parameters $(\theta, \lambda, \bar{\mu})$ and a smooth enough family $\left(V^{\varepsilon}, \eta^{\varepsilon}\right)$ consistent with the system $S_{\theta, \lambda, \mu} \in S$ and defined on $\left[0, \frac{T}{\varepsilon}\right]$ for some $T>0$. If $V^{\varepsilon}$ is nearly irrotationnal in the sense that curl $V^{\varepsilon}=O(\varepsilon)$, then the system $S_{\underline{\theta}, \underline{\lambda}, \underline{\mu}}^{\prime} \in \Sigma$ admits a unique family of solutions $\left(V_{\Sigma}^{\varepsilon}, \eta_{\Sigma}^{\varepsilon}\right)$ defined on $\left[0, \frac{T}{\varepsilon}\right]$ and with initial conditions $\left(V_{\Sigma, 0}^{\varepsilon}, \eta_{\Sigma, 0}^{\varepsilon}\right)$ defined as

$$
\left(V_{\Sigma, 0}^{\varepsilon}, \eta_{\Sigma, 0}^{\varepsilon}\right)=\left(\widetilde{V}_{0}^{\varepsilon}\left(1+\frac{\varepsilon}{2} \eta^{\varepsilon}(t=0)\right), \eta^{\varepsilon}(t=0)\right)
$$

with

$$
\widetilde{V}_{0}^{\varepsilon}=\left(1-\frac{\varepsilon}{2}\left(1-\underline{\theta}^{2}\right) \Delta\right)^{-1}\left(1-\frac{\varepsilon}{2}\left(1-\theta^{2}\right) \Delta\right) V^{\varepsilon}(t=0) .
$$

Moreover, there exists $\varepsilon_{0}>0$ such that for all $0<\varepsilon<\varepsilon_{0}$,

$$
\begin{array}{r}
\left|V^{\varepsilon}-\left(1-\frac{\varepsilon}{2}\left(1-\theta^{2}\right) \Delta\right)^{-1}\left(1-\frac{\varepsilon}{2}\left(1-\underline{\theta}^{2}\right) \Delta\right)\left(V_{\Sigma}^{\varepsilon}\left(1-\frac{\varepsilon}{2} \eta_{\Sigma}^{\varepsilon}\right)\right)\right|_{L^{\infty}\left(0, t ; H^{s}\left(\mathbb{R}^{d}\right)\right)} \\
+\left|\eta^{\varepsilon}-\eta_{\Sigma}^{\varepsilon}\right|_{L^{\infty}\left(0, t ; H^{s}\left(\mathbb{R}^{d}\right)\right)} \leq C \varepsilon^{2} t,
\end{array}
$$

for all $t \in\left[0, \frac{T}{\varepsilon}\right]$.

Proof.

From Prop. 2.1, it follows that $\left(\widetilde{V}^{\varepsilon}, \widetilde{\eta}^{\varepsilon}\right)$, as defined in the proof of Cor. 3.1, is consistent with $S_{\underline{\theta}, \underline{\lambda}, \underline{\mu}} \in S$. From Prop. 2.2, we then deduce that $\left(\widetilde{\widetilde{V^{\varepsilon}}}, \widetilde{\eta^{\varepsilon}}\right)$ defined as

$$
\widetilde{\widetilde{V^{\varepsilon}}}=\widetilde{V}\left(1+\frac{\varepsilon}{2} \widetilde{\eta}\right), \quad \widetilde{\widetilde{\eta^{\varepsilon}}}=\widetilde{\eta},
$$

is consistent with $S_{\underline{\theta}, \underline{\lambda}, \mu}^{\prime} \in \Sigma$.

From Prop. 2.4 one can deduce the existence of $\left(V_{\Sigma}^{\varepsilon}, \eta_{\Sigma}^{\varepsilon}\right)$ and from Th. 3.1, the error estimate between $\left(\widetilde{V^{\varepsilon}}, \widetilde{\eta^{\varepsilon}}\right)$ and $\left(V_{\Sigma}^{\varepsilon}, \eta_{\Sigma}^{\varepsilon}\right)$. Inverting the nonlinear change of variables (which is possible for $\varepsilon$ small enough) and then the pseudo-differential one then yields the result. 
Remark 3.1 The irrotationality condition imposed in Cor. 3.2 is not necessary in $1-D$ since in that case, the non-linear change of variables symmetrizes the hyperbolic part of the Boussinesq system without using it (as seen in Section 2.2).

\subsection{The main result.}

The aim of this section is to state and prove the theorem concerning the approximation of the solution to the Euler equations. We chose to work here with a formulation of the Euler equations alternative to (1.1)-(1.4). This new formulation reads as a system of PDE coupling the height $\eta$ of the free surface to $V=\nabla \psi$, where $\psi$ denotes the values of the potential at the free surface, $\psi(t, X)=\phi(t, X, 1+\varepsilon \eta)$. This new formulation, which involves a DirichletNeumann operator $G_{\varepsilon}$, is derived in Section 4.1. It reads

$$
\partial_{t} V-\varepsilon \nabla\left(\partial_{t} \eta G_{\varepsilon}(\eta) \psi\right)+\nabla \eta+\frac{\varepsilon}{2} \nabla\left(\left|V-\varepsilon \nabla \eta G_{\varepsilon}(\eta) \psi\right|^{2}\right)+\nabla\left|G_{\varepsilon}(\eta) \psi\right|^{2}=0,
$$

for $X \in \mathbb{R}^{d}$ and $t>0$ and

$$
\partial_{t} \eta+\varepsilon \nabla \eta \cdot\left(V-\varepsilon \nabla \eta G_{\varepsilon}(\eta) \psi\right)=\frac{1}{\varepsilon} \nabla\left(G_{\varepsilon}(\eta) \psi\right),
$$

where $V=\nabla \psi, \psi(t, X)=\psi(t, X, 1+\varepsilon \eta)$ and $G_{\varepsilon}(\eta) \psi$ is given by

$$
G_{\varepsilon}(\eta) \psi=\partial_{z} \phi(t, X, 1+\varepsilon \eta)
$$

with

$$
\left\{\begin{array}{l}
\varepsilon \Delta \phi+\partial_{z}^{2} \phi=0, \quad X \in \mathbb{R}^{d}, \quad 0<z<1+\varepsilon \eta \\
\partial_{z} \phi=0 \text { at } z=0, \quad X \in \mathbb{R}^{d} \\
\phi(X, 1+\varepsilon \eta)=\psi(t, X) .
\end{array}\right.
$$

If $\left(V^{\varepsilon}, \eta^{\varepsilon}\right)$ is a solution to (3.5)-(3.6) with initial data $\left(V_{0}^{\varepsilon}, \eta_{0}^{\varepsilon}\right)$, we construct what we call an approximate solution $\left(V_{a p p}^{\varepsilon}, \eta_{a p p}^{\varepsilon}\right)$ to (3.5)-(3.6) as follows. First, one considers $\left({\widetilde{V_{0}}}^{\varepsilon},{\widetilde{\eta_{0}}}^{\varepsilon}\right)$ given by

$$
\widetilde{V}_{0}^{\varepsilon}=\left(1-\frac{\varepsilon}{2}\left(1-\theta^{2}\right) \Delta\right)^{-1} V_{0}^{\varepsilon}, \quad \widetilde{\eta}_{0}^{\varepsilon}=\eta_{0}^{\varepsilon} .
$$

Then take $\left(V_{\Sigma, 0}^{\varepsilon}, \eta_{\Sigma, 0}^{\varepsilon}\right)$ given by:

$$
\left(V_{\Sigma, 0}^{\varepsilon}, \eta_{\Sigma, 0}^{\varepsilon}\right)=\left(\widetilde{V}_{0}^{\varepsilon}\left(1+\frac{\varepsilon}{2} \eta_{0}^{\varepsilon}\right), \eta_{0}^{\varepsilon}\right)
$$

We now choose the parameters $(\underline{\theta}, \underline{\lambda}, \underline{\mu})$ such that the system $S_{\underline{\theta}, \underline{\lambda}, \underline{\mu}}^{\prime}$ belongs to the class $\Sigma$ (i.e. is completely symmetric); in other words, we choose the 
parameters in such a way that (3.1)-(3.2)-(3.3) are satisfied. Let $\left(V_{\Sigma}^{\varepsilon}, \eta_{\Sigma}^{\varepsilon}\right)$ be its solution with initial data $\left(V_{\Sigma}^{\varepsilon}, \eta_{\Sigma}^{\varepsilon}\right)(t=0)=\left(V_{\Sigma, 0}^{\varepsilon}, \eta_{\Sigma, 0}^{\varepsilon}\right)$ which exists and is bounded with respect to $\varepsilon$ in $L^{\infty}\left(0, \frac{T}{\varepsilon} ; H^{s}\right)$ for some $T>0$ by Prop. 2.4.

From this family of solutions of the symmetric system $S_{\underline{\theta}, \underline{\lambda}, \underline{\mu}}^{\prime}$, we obtain our approximate solution to the Euler equations by inverting approximately the nonlinear change of variables, and then the pseudo-differential one, which gives

$$
\left\{\begin{array}{l}
V_{a p p}^{\varepsilon}=\left(1-\frac{\varepsilon}{2}\left(1-\underline{\theta}^{2}\right) \Delta\right)\left[V_{\Sigma}^{\varepsilon}\left(1-\frac{\varepsilon}{2} \eta_{\Sigma}^{\varepsilon}\right)\right], \\
\eta_{\text {app }}^{\varepsilon}=\eta_{\Sigma}^{\varepsilon} .
\end{array}\right.
$$

Theorem 3.2 One dimensional case.

Let $s \geq 0$ and $\left(v_{0}^{\varepsilon}, \eta_{0}^{\varepsilon}\right)$ be a bounded family of $H^{\sigma}(\mathbb{R})^{2}(\sigma \geq s$ large enough). There exist $T>0$ and $\varepsilon_{0}>0$ such that the following holds. There exists a unique solution $\left(v^{\varepsilon}, \eta^{\varepsilon}\right)$ to the Euler equations (3.5)-(3.6) with initial conditions $\left(v_{0}^{\varepsilon}, \eta_{0}^{\varepsilon}\right)$ and one has, for all $0<\varepsilon<\varepsilon_{0}$,

$$
\left|v^{\varepsilon}-v_{a p p}^{\varepsilon}\right|_{L^{\infty}\left(0, t ; H^{s}\right)}+\left|\eta^{\varepsilon}-\eta_{a p p}^{\varepsilon}\right|_{L^{\infty}\left(0, t ; H^{s}\right)} \leq c \varepsilon^{2} t \text { for all } t \in\left[0, \frac{T}{\varepsilon}\right],
$$

where $\left(v_{\text {app }}^{\varepsilon}, \eta_{\text {app }}^{\varepsilon}\right)$ is given by (3.9).

Theorem 3.3 Two dimensional case.

Let $s \geq 0$ and $\left(V_{0}^{\varepsilon}, \eta_{0}^{\varepsilon}\right)$ be a bounded family of $H^{\sigma}\left(\mathbb{R}^{2}\right)^{2}$ ( $\sigma \geq s$ large enough) such that curl $V_{0}^{\varepsilon}=0$. Let $\left(V^{\varepsilon}, \eta^{\varepsilon}\right)$ be a family of solutions to the Euler equations (3.5)-(3.6) with initial conditions $\left(V_{0}^{\varepsilon}, \eta_{0}^{\varepsilon}\right)$ and bounded with respect to $\varepsilon$ in $W^{1, \infty}\left(0, \frac{T}{\varepsilon} ; H^{\sigma}\left(\mathbb{R}^{2}\right)\right)$. Then, for $\varepsilon$ small enough,

$$
\left|V^{\varepsilon}-V_{a p p}^{\varepsilon}\right|_{L^{\infty}\left(0, t ; H^{s}\right)}+\left|\eta^{\varepsilon}-\eta_{\text {app }}^{\varepsilon}\right|_{L^{\infty}\left(0, t ; H^{s}\right)} \leq c \varepsilon^{2} t \text { for all } t \in\left[0, \frac{T}{\varepsilon}\right],
$$

where $\left(V_{\text {app }}^{\varepsilon}, \eta_{\text {app }}^{\varepsilon}\right)$ is given by (3.9).

\section{Proof.}

In the one-dimensional case, the existence part follows from W. Craig [11] or G. Schneider-E. Wayne [19].

The key point of the proof is that the solutions to the Euler system are consistent with the Boussinesq system (2.1). This fact is proved in Th. 4.2. Since this latter system belongs to the class $S$ (it can be written under the form $S_{\theta, \lambda, \mu}$ with $\theta=\lambda=1$ ), the results of both theorems can be deduced from Cor. 3.2 .

\section{Comments:}

1. This result is a true convergence result with improved error bounds and 
not only a consistency result. The available error estimates for KdV-type decoupled systems like in [19] lead only -with the scaling used here- to errors like $O\left(\varepsilon^{1 / 4}\right)$. We improve this result in Section 5 by proving that the error estimate for $K d V$-type uncoupled approximations is $O(\varepsilon)$, and show that this estimate is sharp. It is clear that for short times, the approximation given in Ths. 3.2 and 3.3 is more precise. Moreover, both theorems remain valid in the periodic framework, which is not the case for uncoupled approximations.

2. Among all the systems of the class $S$ and $S^{\prime}$, only those belonging to the class $\Sigma$ are well understood in terms of the Cauchy problem on a time interval of size $\left[0, O\left(\frac{1}{\varepsilon}\right)\right]$. This is why we constructed our approximate solution $\left(V_{a p p}^{\varepsilon}, \eta_{\text {app }}^{\varepsilon}\right)$ in terms of the solution $\left(V_{\Sigma}^{\varepsilon}, \eta_{\Sigma}^{\varepsilon}\right)$ of such a symmetric system $S_{\theta, \lambda, \mu}^{\prime}$. However, an approximate solution can be constructed from any family of solutions consistent with one of the systems of $S$, or $S^{\prime}$ :

If $\left(V_{1}^{\varepsilon}, \eta_{1}^{\varepsilon}\right)$ is consistent with $S_{\theta, \lambda, \mu}$ and coincides with the solution $\left(V^{\varepsilon}, \eta^{\varepsilon}\right)$ of the Euler equations at $t=0$, define $\left(V_{a p p, 1}^{\varepsilon}, \eta_{a p p, 1}^{\varepsilon}\right)$ as

$$
V_{a p p, 1}^{\varepsilon}=\left(1-\frac{\varepsilon}{2}\left(1-\theta^{2}\right) \Delta\right) V_{1}^{\varepsilon}, \quad \eta_{a p p, 1}^{\varepsilon}=\eta_{1}^{\varepsilon} .
$$

From Prop. 2.1, $\left(V_{a p p, 1}^{\varepsilon}, \eta_{a p p, 1}^{\varepsilon}\right)$ is consistent with the Boussinesq system (2.1). Therefore, thanks to Cor. 3.2, one can replace $\left(V^{\varepsilon}, \eta^{\varepsilon}\right)$ by $\left(V_{a p p, 1}^{\varepsilon}, \eta_{a p p, 1}^{\varepsilon}\right)$ in the statement of Ths. 3.1-3.2. This result, together with Ths. 3.1-3.2 yields the following corollary by a triangular inequality.

Corollary 3.3 If $\left(V^{\varepsilon}, \eta^{\varepsilon}\right)$, defined on $\left[0, \frac{T}{\varepsilon}\right]$ for some $T>0$, solves the Euler equations and if curl $V^{\varepsilon}(t=0)=0$ then

$$
\left|V^{\varepsilon}-V_{a p p, 1}^{\varepsilon}\right|_{L^{\infty}\left(0, t ; H^{s}\right)}+\left|\eta^{\varepsilon}-\eta_{a p p, 1}^{\varepsilon}\right|_{L^{\infty}\left(0, t ; H^{s}\right)} \leq c \varepsilon^{2} t \text { for all } t \in\left[0, \frac{T}{\varepsilon}\right] .
$$

Similarly, if $\left(V_{2}^{\varepsilon}, \eta_{2}^{\varepsilon}\right)$ is consistent with $S_{\theta, \lambda, \mu}^{\prime}$ and coincides with the solution $\left(V^{\varepsilon}, \eta^{\varepsilon}\right)$ of the Euler equations at $t=0$, define $\left(V_{a p p, 2}^{\varepsilon}, \eta_{a p p, 2}^{\varepsilon}\right)$ as

$$
V_{a p p, 2}^{\varepsilon}=\left(1-\frac{\varepsilon}{2}\left(1-\theta^{2}\right) \Delta\right)\left(V_{2}^{\varepsilon}\left(1-\frac{\varepsilon}{2} \eta_{2}^{\varepsilon}\right)\right), \quad \eta_{a p p, 2}^{\varepsilon}=\eta_{2}^{\varepsilon} .
$$

With the same method as above, one obtains:

Corollary 3.4 If $\left(V^{\varepsilon}, \eta^{\varepsilon}\right)$, defined on $\left[0, \frac{T}{\varepsilon}\right]$ for some $T>0$, solves the Euler equations and if $\operatorname{curl} V^{\varepsilon}(t=0)=0$ then

$$
\left|V^{\varepsilon}-V_{a p p, 2}^{\varepsilon}\right|_{L^{\infty}\left(0, t ; H^{s}\right)}+\left|\eta^{\varepsilon}-\eta_{a p p, 2}^{\varepsilon}\right|_{L^{\infty}\left(0, t ; H^{s}\right)} \leq c \varepsilon^{2} t \text { for all } t \in\left[0, \frac{T}{\varepsilon}\right] .
$$

3. It follows from Cor. 3.3-3.4 that all the formal approximate systems of the class $S$ of Bona et al. and of the new class $S^{\prime}$ are justified rigorously. This can sometimes be spectacular: the historical Boussinesq system is ill-posed, but nonetheless, we have a convergence result! More precisely, we can prove that any family of solutions to the Euler equations existing over times $O(1 / \varepsilon)$, is well approximated as $\varepsilon \rightarrow 0$ by any family of functions consistent with the Boussinesq system: 
Theorem 3.4 Let $\left(V^{\varepsilon}, \eta^{\varepsilon}\right)$ and $\left(V_{\text {Bous }}^{\varepsilon}, \eta_{\text {Bous }}^{\varepsilon}\right)$ be two bounded family of smooth enough irrotational functions defined on $\left[0, \frac{T}{\varepsilon}\right]$ for some $T>0$, and consistent with the Euler equations and the Boussinesq system (2.1) respectively, then

$$
\left|V^{\varepsilon}-V_{\text {Bous }}^{\varepsilon}\right|_{L^{\infty}\left(0, t ; H^{s}\right)}+\left|\eta^{\varepsilon}-\eta_{\text {Bous }}^{\varepsilon}\right|_{L^{\infty}\left(0, t ; H^{s}\right)} \leq c \varepsilon^{2} t \text { for all } t \in\left[0, \frac{T}{\varepsilon}\right] .
$$

4. Note that the way we use to prove the error estimates is quite unusual. Indeed, we in fact consider that the solution to the Euler equation (that is the "exact solution") is an approximate solution to the system $\Sigma_{\theta, \lambda, \mu}$ that we consider (that is the approximate system). And we perform the error estimate on this completely symmetric system which is the center of the analysis.

5. Another approach to improve the error estimates of the uncoupled $\mathrm{KdV}$ approximation for water-waves consists in computing the next order terms in the BKW expansion. This method, studied by Wayne and Wright [20] for a model problem (which is the Boussinesq equation) has the advantage of giving an $O\left(\varepsilon^{2}\right)$ error term, but cannot be extended to the periodic case.

\section{Consistency of the Euler equations with the Boussinesq system}

\subsection{Statement of the problem}

This aim of this part is to prove the consistency of any solution to the Euler equations with the asymptotic Boussisnesq system. For convenience, let us recall Eqs. (1.1)-(1.4), which are the Euler equations in the long-wave, small amplitude scaling, with Stokes number equal to 1,

$$
\begin{array}{rlrl}
\varepsilon \Delta \phi+\partial_{z}^{2} \phi & =0 & & 0 \leq z \leq 1+\varepsilon \eta, \\
\partial_{z} \phi & =0 & & \text { at } z=0, \\
\partial_{t} \phi+\frac{1}{2}\left(\varepsilon|\nabla \phi|^{2}+\left|\partial_{z} \phi\right|^{2}\right)+\eta & =0 & & \text { at } z=1+\varepsilon \eta, \\
\partial_{t} \eta+\varepsilon \nabla \phi \cdot \nabla \eta=\frac{1}{\varepsilon} \partial_{z} \phi & & \text { at } z=1+\varepsilon \eta,
\end{array}
$$

where $\Delta$ and $\nabla$ denote the usual Laplace and gradient operators in transverse variable $X \in \mathbb{R}^{d}, X=(x, y)$ if $d=2$ and $X=x$ if $d=1$. By the earlier works of W. Craig [11] and Schneider-Wayne [19], one knows that for $d=1$ there exists a smooth solution to (4.1)-(4.4) defined on $\left[0, \frac{T}{\varepsilon}\right]$ bounded (with respect to $\varepsilon)$ in $W^{k, \infty}\left(0, \frac{T}{\varepsilon} ; H^{s}(\mathbb{R})\right)$ for $k$ and $s$ large enough. Following [12], we take $\psi_{\varepsilon}(t, X):=\phi(t, X, 1+\varepsilon \eta(t, X))$ as new unknown; this quantity is the velocity potential at the free surface. As usual, see e.g. [17] [12] [13] [21] [19], we write the equations satisfied by $\eta$ and $\psi_{\varepsilon}$. To this end, we need to use the Dirichlet to Neumann operator which basically expresses the normal 
velocity at the free surface in terms of the value of the potential at the free surface. Since the normal velocity can be deduced from $\partial_{z} \phi$, we consider in this paper the operator which maps $\psi$ to $\left.\partial_{z} \phi\right|_{z=1+\varepsilon \eta}$. More precisely, for any $f \in\left(C^{1} \cap W^{1, \infty}\right)\left(\mathbb{R}^{d}\right)$ and for any $\varepsilon$ such that $0<1-\varepsilon|f|_{\infty}$, we define the operator $G_{\varepsilon}(f)$, which acts on $H^{3 / 2}\left(\mathbb{R}^{d}\right)$ with values in $H^{1 / 2}\left(\mathbb{R}^{d}\right)$ as

$$
G_{\varepsilon}(f) g=\partial_{z} u(X, 1+\varepsilon f),
$$

where $u$ is the solution to

$$
\begin{array}{rlrl}
\varepsilon \Delta u+\partial_{z}^{2} u=0 & & 0 \leq z \leq 1+\varepsilon f, \quad X \in \mathbb{R}^{d} \\
\partial_{z} u=0 & & \text { at } z=0, \quad X \in \mathbb{R}^{d} \\
u(X, 1+\varepsilon f) & =g & & X \in \mathbb{R}^{d} .
\end{array}
$$

Before rewriting Eqs. (4.1)-(4.4) using this operator, we need to compute the derivatives of $\phi$ in terms of $\psi$ and $\eta$. Simple computations yield

$$
\begin{aligned}
\left.\partial_{t} \phi\right|_{z=1+\varepsilon \eta} & =\partial_{t} \psi-\varepsilon \partial_{t} \eta G_{\varepsilon}(\eta) \psi \\
\left.\nabla \phi\right|_{1+\varepsilon \eta} & =\nabla \psi-\varepsilon \nabla \eta G_{\varepsilon}(\eta) \psi .
\end{aligned}
$$

Thanks to (4.9)-(4.10), we can rewrite (4.1)-(4.4) as

$$
\begin{array}{r}
\partial_{t} \psi-\varepsilon \partial_{t} \eta G_{\varepsilon}(\eta) \psi+\eta+\frac{\varepsilon}{2}\left|\nabla \psi-\varepsilon \nabla \eta G_{\varepsilon}(\eta) \psi\right|^{2}+\frac{1}{2}\left|G_{\varepsilon}(\eta) \psi\right|^{2}=0, \\
\partial_{t} \eta+\varepsilon \nabla \eta \cdot\left[\nabla \psi-\varepsilon \nabla \eta G_{\varepsilon}(\eta) \psi\right]=\frac{1}{\varepsilon} G_{\varepsilon}(\eta) \psi,
\end{array}
$$

with $X \in \mathbb{R}^{d}$ and $t \in\left[0, \frac{T}{\varepsilon}\right]$.

The next step is to obtain an asymptotic expansion of the operator $G_{\varepsilon}$ as $\varepsilon \rightarrow 0$. This is the goal of the next theorem.

Theorem 4.1 For any $(f, g) \in H^{2}\left(\mathbb{R}^{d}\right)^{2}$ define $G_{1}(f) g=-\Delta g$ and $G_{2}(f) g=$ $-\frac{1}{3} \Delta^{2} g-f \Delta g$.

For any $s \in \mathbb{N}$ there exists $\sigma \in \mathbb{N}, \sigma \geq s$ and $\varepsilon_{0}>0$ such that $\forall \varepsilon>0, \varepsilon<\varepsilon_{0}$, if $(f, g) \in H^{\sigma}\left(\mathbb{R}^{d}\right)^{2}$, one has

$$
\left|G_{\varepsilon}(f) g-\varepsilon G_{1}(f) g-\varepsilon^{2} G_{2}(f) g\right|_{H^{s}\left(\mathbb{R}^{d}\right)} \leq \varepsilon^{3} C\left(|f|_{H^{\sigma}},|g|_{H^{\sigma}}\right),
$$

where $C$ is a continuous function of its arguments.

Remark 4.1 This theorem could certainly be obtained using the estimates of the Dirichlet to Neumann operators in the works of Craig [11] and SchneiderWayne [19]. However our proof has an interest in itself since it is simpler and uses directly the elliptic equations. We postpone it to Section 4.3. Note also that a similar approach is used in [18]. 


\subsection{Asymptotic expansion of the solutions of the Euler equations}

The aim of this section is to give an asymptotic expansion to the solutions of (4.11)-(4.12). This is done in the following theorem, which says that any solution of (4.11)-(4.12) is consistent with the Boussinesq system.

Theorem 4.2 Let $s \geq 0$. Let $\left(\psi^{\varepsilon}, \eta^{\varepsilon}\right)$ be a solution to Eqs. (4.11)-(4.12). There exists $\sigma \in \mathbb{N}$ such that if $\left(\psi^{\varepsilon}, \eta^{\varepsilon}\right)$ is bounded in $W^{1, \infty}\left(0, \frac{T}{\varepsilon} ; H^{\sigma}\left(\mathbb{R}^{d}\right)\right)$, then

$$
\begin{array}{r}
\partial_{t} V^{\varepsilon}+\nabla \eta^{\varepsilon}+\frac{\varepsilon}{2} \nabla\left(\left|V^{\varepsilon}\right|^{2}\right)=\varepsilon^{2} r_{1}^{\varepsilon} \\
\partial_{t} \eta^{\varepsilon}+\nabla \cdot V^{\varepsilon}+\varepsilon\left(\nabla \cdot\left(\eta^{\varepsilon} V^{\varepsilon}\right)+\frac{1}{3} \Delta \nabla \cdot V^{\varepsilon}\right)=\varepsilon^{2} r_{2}^{\varepsilon},
\end{array}
$$

where $V^{\varepsilon}:=\nabla \psi^{\varepsilon}$, and $r_{1}^{\varepsilon}, r_{2}^{\varepsilon}$ are bounded in $L^{\infty}\left(0, \frac{T}{\varepsilon} ; H^{s}\left(\mathbb{R}^{d}\right)\right)$.

Remark 4.2 From previous works [11] [19] it is known that when $d=1$, equations (4.11)-(4.12) are well-posed. Therefore the assumption made in Th. 4.2 on the existence and regularity of $\left(\psi^{\varepsilon}, \eta^{\varepsilon}\right)$ reduces to a simple assumption of regularity on the initial conditions $\left(\psi_{0}, \eta_{0}\right) \in H^{\sigma_{0}}(\mathbb{R})$ taken for (4.11)-(4.12).

Proof.

Let $\left(\psi^{\varepsilon}, \eta^{\varepsilon}\right)$ be as in the statement of the theorem. Using Th. 4.1, one has

$$
G_{\varepsilon}\left(\eta^{\varepsilon}\right) \psi^{\varepsilon}+\varepsilon \Delta \psi^{\varepsilon}+\varepsilon^{2}\left(\frac{1}{3} \Delta^{2} \psi^{\varepsilon}+\eta^{\varepsilon} \Delta \psi^{\varepsilon}\right)=O\left(\varepsilon^{3}\right)
$$

where the $O\left(\varepsilon^{3}\right)$ error term is taken in $L^{\infty}\left(0, \frac{T}{\varepsilon} ; H^{s}\left(\mathbb{R}^{d}\right)\right)$ norm.

Plugging (4.13) into (4.11) and keeping only the terms of order $O(\varepsilon)$ yields

$$
\partial_{t} \psi^{\varepsilon}+\eta^{\varepsilon}+\frac{\varepsilon}{2}\left|\nabla \psi^{\varepsilon}\right|^{2}=O\left(\varepsilon^{2}\right), \quad X \in \mathbb{R}^{d}, \quad t \in\left[0, \frac{T}{\varepsilon}\right] .
$$

Plugging (4.13) into (4.12) leads to

$$
\partial_{t} \eta^{\varepsilon}+\varepsilon \nabla \eta^{\varepsilon} \cdot \nabla \psi^{\varepsilon}=-\Delta \psi^{\varepsilon}-\varepsilon\left(\frac{1}{3} \Delta^{2} \psi^{\varepsilon}+\eta^{\varepsilon} \Delta \psi^{\varepsilon}\right)+O\left(\varepsilon^{2}\right),
$$

for $X \in \mathbb{R}^{d}$ and $t \in\left[0, \frac{T}{\varepsilon}\right]$.

Introducing $V^{\varepsilon}=\nabla \psi^{\varepsilon}$, one gets, taking the gradient of (4.14),

$$
\begin{array}{r}
\partial_{t} V^{\varepsilon}+\nabla \eta^{\varepsilon}+\frac{\varepsilon}{2} \nabla\left(\left|V^{\varepsilon}\right|^{2}\right)=O\left(\varepsilon^{2}\right) \\
\partial_{t} \eta^{\varepsilon}+\nabla \cdot V^{\varepsilon}+\varepsilon\left(\nabla \cdot\left(\eta^{\varepsilon} V^{\varepsilon}\right)+\frac{1}{3} \Delta \nabla \cdot V^{\varepsilon}\right)=O\left(\varepsilon^{2}\right) .
\end{array}
$$

Note that the error terms $O\left(\varepsilon^{2}\right)$ are in $L^{\infty}\left(0, \frac{T}{\varepsilon} ; H^{s}\left(\mathbb{R}^{d}\right)\right)$ for any chosen $s$, provided that $\sigma$ is large enough. 


\subsection{Expansion of $G_{\varepsilon}$}

This section is devoted to the proof of Th. 4.1. Recall that we are concerned with the operator $G_{\varepsilon}$ defined by (4.5)-(4.8). By a change of variable, we can work on an horizontal strip: let $\widetilde{u}$ be defined on $\mathcal{S}:=\left\{X \in \mathbb{R}^{d}, z \in[0,1]\right\}$ by

$$
\widetilde{u}(X, z):=u(X, z(1+\varepsilon f)),
$$

where $u$ and $f$ are as in (4.5)-(4.8). It follows immediately that for all $X \in \mathbb{R}^{d}$, for all $z \in[0,1+\varepsilon f]$, one has $u(X, z)=\widetilde{u}\left(X, \frac{z}{1+\varepsilon f}\right)$. We now deduce from (4.5)-(4.8) the equations satisfied by $\widetilde{u}$. Eqs. (4.7), (4.8) and (4.5) yield respectively

$$
\begin{array}{rr}
\partial_{z} \widetilde{u}(X, 0)=0 & X \in \mathbb{R}^{d}, \\
\widetilde{u}(X, 1)=g(X) & X \in \mathbb{R}^{d}, \\
G_{\varepsilon}(f)(g)=\frac{1}{1+\varepsilon f} \partial_{z} \widetilde{u}(X, 1) & X \in \mathbb{R}^{d} .
\end{array}
$$

In order to use (4.6) we must compute $\partial_{z}^{2} u$ and $\Delta u$ in terms of $\widetilde{u}$. Introducing $\chi_{\varepsilon}:=\frac{1}{1+\varepsilon f}$, one obtains

$$
\begin{aligned}
\partial_{z}^{2} u & =\chi_{\varepsilon}^{2} \partial_{z}^{2} \widetilde{u}, \\
\Delta u & =\Delta \widetilde{u}+z \Delta \chi_{\varepsilon} \partial_{z} \widetilde{u}+2 z \nabla \partial_{z} \widetilde{u} \cdot \nabla \chi_{\varepsilon}+z^{2}\left|\nabla \chi_{\varepsilon}\right|^{2} \partial_{z}^{2} \widetilde{u},
\end{aligned}
$$

and Eq. (4.6) therefore reads

$$
\varepsilon \Delta \widetilde{u}+\left(\chi_{\varepsilon}^{2}+\varepsilon \widetilde{u}^{2}\left|\nabla \chi_{\varepsilon}\right|^{2}\right) \partial_{z}^{2} \widetilde{u}+2 \varepsilon z \nabla \chi_{\varepsilon} \cdot \nabla \partial_{z} \widetilde{u}+\varepsilon z \Delta \chi_{\varepsilon} \partial_{z} \widetilde{u}=0 .
$$

Let us now define

$$
\begin{aligned}
& \widetilde{u}_{0}:=g, \\
& \widetilde{u}_{1}:=-\frac{\left(z^{2}-1\right)}{2} \Delta g, \\
& \widetilde{u}_{2}:=\frac{\Delta^{2} g}{4}\left(\frac{z^{4}}{6}-z^{2}+\frac{5}{6}\right)-f \Delta g\left(z^{2}-1\right) .
\end{aligned}
$$

In order to prove Th. 4.1, we prove the following proposition,

Proposition 4.1 For any $s \in \mathbb{N}$, there exists $\sigma \in \mathbb{N}$ and $\varepsilon_{0}>0$ such that for all $\varepsilon>0, \varepsilon<\varepsilon_{0}$, and for all $(f, g) \in H^{\sigma}\left(\mathbb{R}^{d}\right)^{2}$, the solution $\widetilde{u}$ of (4.19), (4.20) and (4.22) satisfies

$$
\widetilde{u}-\left(\widetilde{u}_{0}+\varepsilon \widetilde{u}_{1}+\varepsilon^{2} \widetilde{u}_{2}\right)=O\left(\varepsilon^{3}\right) \quad \text { in } H^{s}(\mathcal{S}),
$$

where $\widetilde{u}_{0}, \widetilde{u}_{1}$ and $\widetilde{u}_{2}$ are given in (4.23). 


\section{Proof.}

In order to prove this proposition, we proceed as follows:

i. In a first step, we construct an approximate solution to (4.19), (4.20) and (4.22), up to order $O\left(\varepsilon^{3}\right)$.

ii. In a second step, we perform energy estimate on the difference between the exact and the approximate solutions.

Step 1: Construction of the approximate solution.

The energy estimates we will have to use in Step 2 use the principal part of (4.22), which reads $\varepsilon \Delta \widetilde{u}+\left(\chi_{\varepsilon}^{2}+\varepsilon z^{2}\left|\nabla \chi_{\varepsilon}\right|^{2}\right) \partial_{z}^{2} \widetilde{u}$. Because of the coefficient $\varepsilon$ in front of $\Delta \widetilde{u}$, the error estimate on $X$ derivatives are one order worse (in terms of $\varepsilon$ ) than $z$-derivatives. This is the reason why we need an approximate solution to one order further than $O\left(\varepsilon^{2}\right)$, i.e. to order $O\left(\varepsilon^{3}\right)$. We therefore make the following ansatz for the approximate solution,

$$
\widetilde{u}_{a}=\widetilde{u}_{0}+\varepsilon \widetilde{u}_{1}+\varepsilon^{2} \widetilde{u}_{2}+\varepsilon^{3} \widetilde{u}_{3}
$$

and plug it into (4.22) assuming that $\partial_{z} \widetilde{u}_{i}=0$ at $z=0$ for $i=0 \ldots 3$, that $\widetilde{u}_{0}=g$ at $z=1$ and $\widetilde{u}_{i}=0$ at $z=1$ for $i=1 \ldots 3$.

At order $\varepsilon^{0}$, we find $\partial_{z}^{2} \widetilde{u}_{0}=0$, which with the boundary conditions on $\widetilde{u}_{0}$ yields $\widetilde{u}_{0}=g$.

In order to expand (4.22) into powers of $\varepsilon$ at order 3 , we need to expand $\chi_{\varepsilon}$ and its derivatives as follows,

$$
\begin{aligned}
\chi_{\varepsilon} & =1-\varepsilon f+\varepsilon^{2} f^{2}+O\left(\varepsilon^{3}\right), \\
\left|\chi_{\varepsilon}\right|^{2} & =1-2 \varepsilon f+3 \varepsilon^{2} f^{2}+O\left(\varepsilon^{3}\right), \\
\left|\nabla \chi_{\varepsilon}\right|^{2} & =\varepsilon^{2}|\nabla f|^{2}+O\left(\varepsilon^{3}\right), \\
\Delta \chi_{\varepsilon} & =-\varepsilon \Delta f+O\left(\varepsilon^{2}\right) .
\end{aligned}
$$

Using these expressions and neglecting the terms of order $O\left(\varepsilon^{4}\right)$ and higher in (4.22) gives an equation of the form $\varepsilon P_{1}+\varepsilon^{2} P_{2}+\varepsilon^{3} P_{3}=O\left(\varepsilon^{4}\right)$. Choosing $\widetilde{u}_{1}$, $\widetilde{u}_{2}$ and $\widetilde{u}_{3}$ in order to cancel $P_{1}, P_{2}$ and $P_{3}$ yields three equations. The first one, namely $P_{1}=0$ reads

$$
\Delta \widetilde{u}_{0}+\partial_{z}^{2} \widetilde{u}_{1}=0
$$

the second one, $P_{2}=0$, gives

$$
\Delta \widetilde{u}_{1}+\partial_{z}^{2} \widetilde{u}_{2}-2 f \partial_{z}^{2} \widetilde{u}_{1}=0
$$

and finally $P_{3}=0$ reads

$$
\Delta \widetilde{u}_{2}+\partial_{z}^{2} \widetilde{u}_{3}-2 f \partial_{z}^{2} \widetilde{u}_{2}+3 f^{2} \partial_{z}^{2} \widetilde{u}_{1}-2 z \nabla f \partial_{z} \nabla \widetilde{u}_{1}-z^{2} \Delta f \partial_{z} \widetilde{u}_{1}=0 .
$$

Using the boundary conditions, these equations can be solved; for (4.24), one gets

$$
\widetilde{u}_{1}=-\frac{\Delta g}{2}\left(z^{2}-1\right)
$$


For $\widetilde{u}_{2},(4.25)$ gives $\partial_{z}^{2} \widetilde{u}_{2}=\frac{\Delta^{2} \widetilde{u}_{0}}{2}\left(z^{2}-1\right)-2 f \Delta \widetilde{u}_{0}$, and hence

$$
\widetilde{u}_{2}=\frac{\Delta^{2} g}{4}\left(\frac{z^{4}}{6}-z^{2}+\frac{5}{6}\right)-f \Delta g\left(z^{2}-1\right)
$$

The equation (4.26) gives the value of $\widetilde{u}_{3}$, but since it has no interest, we omit the computation.

Step 2: Error estimates.

We introduce now the difference between the exact and the approximate solutions of (4.22), namely $w:=\widetilde{u}-\widetilde{u}_{a}$. Then $w$ satisfies

$$
\varepsilon \Delta w+\underbrace{\left(\chi_{\varepsilon}^{2}+\varepsilon z^{2}\left|\nabla \chi_{\varepsilon}\right|^{2}\right)}_{:=a_{\varepsilon}(X, z)} \partial_{z}^{2} w+2 \varepsilon \underbrace{z \nabla \chi_{\varepsilon}}_{:=\varepsilon b_{\varepsilon}(X, z)} \cdot \partial_{z} \nabla w+\varepsilon \underbrace{z \Delta \chi_{\varepsilon}}_{:=\varepsilon c_{\varepsilon}(X, z)} \partial_{z} w=O\left(\varepsilon^{4}\right),
$$

and $\partial_{z} w=0$ at $z=0, w=0$ at $z=1$.

Remark that $a_{\varepsilon}, b_{\varepsilon}$ and $c_{\varepsilon}$ are uniformly bounded in $W^{k, \infty}(\mathcal{S}), k$ being possibly as large as one wants to, provided that $\sigma$ is large enough. Moreover, in $W^{k, \infty}(\mathcal{S})$, one has $a_{\varepsilon}=1+O(\varepsilon)$.

We first prove the estimate of Prop. 4.1 for $s=1$ and $s=2$, and then prove the general result by induction.

First multiply (4.29) by $w$ and integrate on the strip $\mathcal{S}=\left\{X \in \mathbb{R}^{d}, z \in[0,1]\right\}$. This yields

$$
\begin{array}{r}
-\varepsilon \int|\nabla w|^{2}-\int a_{\varepsilon}\left|\partial_{z} w\right|^{2}-\int \partial_{z} a_{\varepsilon} \partial_{z} w w-2 \varepsilon^{2} \int b_{\varepsilon} \cdot \nabla w \partial_{z} w \\
-2 \varepsilon^{2} \int \nabla \cdot b_{\varepsilon} \partial_{z} w w+\varepsilon^{2} \int c_{\varepsilon} \partial_{z} w w=\int O\left(\varepsilon^{4}\right) w
\end{array}
$$

where the boundary conditions on $w$ have been used to cancel out the boundary terms in the integration by parts performed with respect to the $z$ variable. Since $a_{\varepsilon}=1+O(\varepsilon)$, the second term of the above equation can easily be controlled, and the third one satisfies

$$
\left|\int \partial_{z} a_{\varepsilon} \partial_{z} w w\right| \leq O(\varepsilon)\left|\partial_{z} w\right||w|
$$

where $|\cdot|$ denotes here the $L^{2}$-norm on $\mathcal{S}$. From Poincaré's inequality, it follows that

$$
\left|\int \partial_{z} a_{\varepsilon} \partial_{z} w w\right| \leq O(\varepsilon)\left|\partial_{z} w\right|^{2}
$$

For the fourth term of the l.h.s. of (4.30), remark that

$$
\begin{aligned}
\left|2 \varepsilon^{2} \int b_{\varepsilon} \cdot \nabla w \partial_{z} w\right| & \leq O\left(\varepsilon^{2}\right)\left|\partial_{z} w\right||\nabla w| \\
& \leq O\left(\varepsilon^{2}\right)\left(\left|\partial_{z} w\right|^{2}+|\nabla w|^{2}\right) .
\end{aligned}
$$


Similarly, the fifth and sixth terms are controlled by

$$
\left|2 \varepsilon^{2} \int \nabla \cdot b_{\varepsilon} \partial_{z} w w+\varepsilon^{2} \int c_{\varepsilon} \partial_{z} w w\right| \leq O\left(\varepsilon^{2}\right)\left|\partial_{z} w\right|^{2},
$$

where Poincaré's inequality has been used once again.

Plugging (4.31)-(4.33) into (4.30) gives therefore

$$
\varepsilon \int|\nabla w|^{2}+\int\left|\partial_{z} w\right|^{2}=O\left(\varepsilon^{4}\right)\left(\int|w|^{2}\right)^{1 / 2},
$$

and hence, by Poincaré's inequality,

$$
\varepsilon \int|\nabla w|^{2}+\int\left|\partial_{z} w\right|^{2}=O\left(\varepsilon^{8}\right)
$$

This last equations yields

$$
\left|\partial_{z} w\right|=O\left(\varepsilon^{4}\right), \quad|\nabla w|=O\left(\varepsilon^{7 / 2}\right),
$$

which proves the proposition for $s=1$.

We now prove it for $s=2$. Taking the $L^{2}$ scalar product of (4.29) with $\Delta w$ yields

$$
\begin{aligned}
\varepsilon \int|\Delta w|^{2}+\int a_{\varepsilon} \partial_{z}^{2} w \Delta w+2 \varepsilon^{2} \int b_{\varepsilon} \cdot \partial_{z} \nabla w \Delta w & +\varepsilon^{2} \int c_{\varepsilon} \partial_{z} w \Delta w \\
& =\int O\left(\varepsilon^{4}\right) \Delta w
\end{aligned}
$$

and taking the $L^{2}$ scalar product of (4.29) with $\partial_{z}^{2} w$ gives

$$
\begin{aligned}
\varepsilon \int \Delta w \partial_{z}^{2} w+\int a_{\varepsilon}\left|\partial_{z}^{2} w\right|^{2}+2 \varepsilon^{2} \int b_{\varepsilon} \cdot \partial_{z} \nabla w \partial_{z}^{2} w+ & \varepsilon^{2} \int c_{\varepsilon} \partial_{z} w \partial_{z}^{2} w \\
& =\int O\left(\varepsilon^{4}\right) \partial_{z}^{2} w
\end{aligned}
$$

We first handle the two terms of the above equations that will give us control of the crossed derivatives, namely $\int a_{\varepsilon} \partial_{z}^{2} w \Delta w$ and $\varepsilon \int \Delta w \partial_{z}^{2} w$. First remark that

$$
\begin{aligned}
\int \Delta w \partial_{z}^{2} w & =-\int \partial_{z} \Delta w \partial_{z} w+\left[\int_{\mathbb{R}^{d}} \Delta w \partial_{z} w\right]_{z=0}^{z=1} \\
& =\int\left|\partial_{z} \nabla w\right|^{2}
\end{aligned}
$$

since the boundary terms are zero, thanks to the boundary conditions satisfied by $w$.

The other term can be treated as

$$
\begin{aligned}
\int a_{\varepsilon} \partial_{z}^{2} w \Delta w & =-\int \partial_{z} a_{\varepsilon} \partial_{z} w \Delta w-\int a_{\varepsilon} \partial_{z} w \partial_{z} \Delta w+\left[\int_{\mathbb{R}^{d}} a_{\varepsilon} \partial_{z} w \Delta w\right]_{z=0}^{z=1} \\
& =-\int \partial_{z} a_{\varepsilon} \partial_{z} w \Delta w+\int \nabla a_{\varepsilon} \partial_{z} w \partial_{z} \nabla w+\int a_{\varepsilon}\left|\partial_{z} \nabla w\right|^{2}
\end{aligned}
$$


Using the fact that $a_{\varepsilon}=1+O(\varepsilon)$ and (4.34), it follows that

$$
\int a_{\varepsilon} \partial_{z}^{2} w \Delta w=O\left(\varepsilon^{5}\right)\left(|\Delta w|+\left|\partial_{z} \nabla w\right|\right)+\int a_{\varepsilon}\left|\partial_{z} \nabla w\right|^{2} .
$$

Then, using (4.37) and (4.38) in (4.35) and (4.36) gives

$$
\begin{aligned}
& \varepsilon \int|\Delta w|^{2}+\int a_{\varepsilon}\left|\partial_{z}^{2} w\right|^{2}+\varepsilon \int\left|\partial_{z} \nabla w\right|^{2}+\int a_{\varepsilon}\left|\partial_{z} \nabla w\right|^{2} \\
& \quad=O\left(\varepsilon^{5}\right)\left(|\Delta w|+\left|\partial_{z} \nabla w\right|\right)+O\left(\varepsilon^{2}\right)\left|\partial_{z} \nabla w\right||\Delta w|+O\left(\varepsilon^{2}\right)\left|\partial_{z} w\right||\Delta w| \\
& \quad+O\left(\varepsilon^{2}\right)\left|\partial_{z} \nabla w\right|\left|\partial_{z}^{2} w\right|+O\left(\varepsilon^{2}\right)\left|\partial_{z} w\right|\left|\partial_{z}^{2} w\right|+O\left(\varepsilon^{4}\right)\left(|\Delta w|+\left|\partial_{z}^{2} w\right|\right) .
\end{aligned}
$$

Multiple use of the identity $a b \leq \frac{1}{2}\left(a^{2}+b^{2}\right)$ and of the fact that $a_{\varepsilon}=1+O(\varepsilon)$ then yields

$$
\varepsilon \int|\Delta w|^{2}+\int\left|\partial_{z}^{2} w\right|^{2}+\int\left|\partial_{z} \nabla w\right|^{2}=O\left(\varepsilon^{8}\right),
$$

which implies the proposition for $s=2$.

We can now turn to prove the general result of the proposition by induction. We assume that for any derivative $\partial$, and for some integer $s \geq 1$, both $\sqrt{\varepsilon}\left|\partial^{s} \partial_{z} w\right|$ and $\left|\partial^{s-1} \partial_{z}^{2} w\right|$ are of size $O\left(\varepsilon^{4}\right)$.

We apply $\partial^{s}$ on (4.29) and take the $L^{2}$ scalar product with $\partial^{s} \partial_{z}^{2} w$, which gives

$$
\begin{array}{r}
\varepsilon \int \Delta \partial^{s} w \partial^{s} \partial_{z}^{2} w+\int \partial^{s}\left(a_{\varepsilon} \partial_{z}^{2} w\right) \partial^{s} \partial_{z}^{2} w+2 \varepsilon^{2} \int \partial^{s}\left(b_{\varepsilon} \partial_{z} \nabla w\right) \partial^{s} \partial_{z}^{2} w \\
+\varepsilon^{2} \int \partial^{s}\left(c_{\varepsilon} \partial_{z} w\right) \partial^{s} \partial_{z}^{2} w=\int O\left(\varepsilon^{4}\right) \partial^{s} \partial_{z}^{2} w .
\end{array}
$$

We now rewrite this equation as follows,

$$
\begin{array}{r}
\varepsilon \int\left|\nabla \partial^{s} \partial_{z} w\right|^{2}+\int\left(\left[\partial^{s}, a_{\varepsilon}\right] \partial_{z}^{2} w\right) \partial_{z}^{2} \partial^{s} w+\int a_{\varepsilon}\left|\partial^{s} \partial_{z}^{2} w\right|^{2} \\
+\varepsilon^{2} \int\left(\left[\partial^{s}, b\right] \partial_{z} \nabla w\right) \partial^{s} \partial_{z}^{2} w+2 \varepsilon^{2} \int b_{\varepsilon} \partial^{s} \partial_{z} \nabla w \partial^{s} \partial_{z}^{2} w \\
+\varepsilon^{2} \int\left(\left[\partial^{s}, c_{\varepsilon}\right] \partial_{z} w\right) \partial^{s} \partial_{z}^{2} w+\varepsilon^{2} \int c_{\varepsilon} \partial^{s} \partial_{z} w \partial^{s} \partial_{z}^{2} w=\int O\left(\varepsilon^{4}\right) \partial^{s} \partial_{z}^{2} w
\end{array}
$$

where $[P, Q]$ denotes the commutator of the operators $P$ and $Q$ :

$$
[P, Q]=P \circ Q-Q \circ P .
$$

We can easily control the terms containing commutators,

$$
\begin{aligned}
&\left|\int\left(\left[\partial^{s}, a_{\varepsilon}\right] \partial_{z}^{2} w\right) \partial_{z}^{2} \partial^{s} w\right| \leq \operatorname{Cst}\left|\partial_{z}^{2} w\right|_{H^{s-1}(\mathcal{S})}\left|\partial_{z}^{2} \partial^{s} w\right| \\
&\left|\varepsilon^{2} \int\left(\left[\partial^{s}, b_{\varepsilon}\right] \partial_{z} \nabla w\right) \partial_{z}^{2} \partial^{s} w\right| \leq \operatorname{Cst} \varepsilon^{2}\left|\partial_{z} w\right|_{H^{s}(\mathcal{S})}\left|\partial^{s} \partial_{z}^{2} w\right| \\
&\left|\varepsilon^{2} \int\left(\left[\partial^{s}, c_{\varepsilon}\right] \partial_{z} w\right) \partial_{z}^{2} \partial^{s} w\right| \leq \operatorname{Cst} \varepsilon^{2}\left|\partial_{z} w\right|_{H^{s-1}(\mathcal{S})}\left|\partial^{s} \partial_{z}^{2} w\right|
\end{aligned}
$$


where we have used $\left|\left[\partial^{s}, a_{\varepsilon}\right] w\right|_{L^{2}} \leq$ Cst $|w|_{H^{s-1}}$. The other terms of (4.39) are controlled by

$$
\begin{aligned}
\left|2 \varepsilon^{2} \int b_{\varepsilon} \partial^{s} \partial_{z} \nabla w \partial^{s} \partial_{z}^{2} w\right| & \leq O\left(\varepsilon^{2}\right)\left|\partial_{z} w\right|_{H^{s+1}}\left|\partial_{z}^{2} w\right|_{H^{s}}, \\
\left|\varepsilon^{2} \int c_{\varepsilon} \partial^{s} \partial_{z} w \partial^{s} \partial_{z}^{2} w\right| & \leq O\left(\varepsilon^{2}\right)\left|\partial_{z} w\right|_{H^{s}}\left|\partial_{z}^{2} w\right|_{H^{s}}
\end{aligned}
$$

Plugging all these estimates into (4.39), one gets

$$
\begin{aligned}
& \varepsilon \int\left|\nabla \partial^{s} \partial_{z} w\right|^{2}+\int\left|\partial^{s} \partial_{z}^{2} w\right|^{2} \\
& \quad \lesssim\left|\partial_{z}^{2} w\right|_{H^{s-1}}\left|\partial_{z}^{2} \partial^{s} w\right|+\varepsilon^{2}\left|\partial_{z} w\right|_{H^{s}}\left|\partial^{s} \partial_{z}^{2} w\right|+\varepsilon^{2}\left|\partial_{z} w\right|_{H^{s-1}}\left|\partial^{s} \partial_{z}^{2} w\right| \\
& \quad+\varepsilon^{2}\left|\partial_{z} w\right|_{H^{s+1}}\left|\partial_{z}^{2} w\right|_{H^{s}}+\varepsilon^{2}\left|\partial_{z} w\right|_{H^{s}}\left|\partial_{z}^{2} w\right|_{H^{s}}+\int O\left(\varepsilon^{4}\right) \partial^{s} \partial_{z}^{2} w
\end{aligned}
$$

where by $\lesssim$, we mean that the r.h.s. should be multiplied by a constant. Recalling that $\sqrt{\varepsilon}\left|\partial_{z} w\right|_{H^{s}}$ and $\left|\partial_{z}^{2} w\right|_{H^{s-1}}$ are of size $O\left(\varepsilon^{4}\right)$, one gets

$$
\varepsilon\left|\partial_{z} w\right|_{H^{s+1}}^{2}+\left|\partial_{z}^{2} w\right|_{H^{s}}^{2}=O\left(\varepsilon^{8}\right),
$$

which completes the induction. The proposition is deduced from the induction property using Poincaré's inequality once again.

We can now conclude the proof of Th. 4.1. We recall that the operator $G_{\varepsilon}(f)(g)$ is given by $(4.21)$ as $G_{\varepsilon}(f)(g)=\frac{1}{1+\varepsilon f} \partial_{z} \widetilde{u}(z=1)$. Therefore, we need to compute $\partial_{z} \widetilde{u}(z=1)$. From Prop. 4.1, we deduce that in $H^{s}\left(\mathbb{R}^{d}\right)$ norm, one has $\left.\partial_{z} \widetilde{u}\right|_{z=1}=\left.\left(\partial_{z} \widetilde{u}_{0}+\varepsilon \partial_{z} \widetilde{u}_{1}+\varepsilon^{2} \partial_{z} \widetilde{u}_{2}\right)\right|_{z=1}+O\left(\varepsilon^{3}\right)$. Using the explicit expressions (4.23) for the $\widetilde{u}_{i}, i=0 \ldots 2$, we finally get

$$
\partial_{z} \widetilde{u}(z=1)=-\varepsilon \Delta g-\frac{\varepsilon^{2}}{3} \Delta^{2} g-2 \varepsilon^{2} f \Delta g+O\left(\varepsilon^{3}\right),
$$

and an easy Taylor expansion of (4.21) therefore yields the Theorem.

\section{The uncoupled approximation}

Many uncoupled models exist for the water-wave equations in the case of one dimensionnal surfaces. Schneider and Wayne [19] proved that the uncoupled $\mathrm{KdV}-\mathrm{KdV}$ approximation is indeed a good model. Our goal here is to justify a whole class of uncoupled models (including the KdV-KdV or BBM-BBM models) obtained formally from the water-wave equations. Moreover, we give sharp error estimates, sensibly better than those of [19], and also comment on the validity of the uncoupled models for the periodic case: in particular, we show that these models are not valid in the periodic setting if one does not make a zero-mass assumption on the initial data. 


\subsection{From the symmetric systems to uncoupled approx- imations}

We construct here approximate solutions to the symmetric systems of the class $\Sigma$, namely,

$$
\left\{\begin{array}{l}
\partial_{t} v+\partial_{x} \eta+\varepsilon\left(\frac{1}{4} \partial_{x} \eta^{2}+\frac{3}{4} \partial_{x} v^{2}+a \partial_{x}^{3} \eta-b \partial_{x}^{2} \partial_{t} v\right)=0 \\
\partial_{t} \eta+\partial_{x} v+\varepsilon\left(\frac{1}{2} \partial_{x}(\eta v)+a \partial_{x}^{3} v-d \partial_{x}^{2} \partial_{t} \eta\right)=0
\end{array}\right.
$$

where $a, b$ and $d$ are as given by (3.3).

We first diagonalize these systems introducing the unknowns $U=v+\eta$ and $N=v-\eta$ so that (5.40) reads

$$
\left\{\begin{aligned}
\partial_{t} U+\partial_{x} U+\quad & \varepsilon\left(\frac{1}{8} \partial_{x}\left(3 U^{2}+N^{2}+2 U N\right)\right. \\
& \left.+a \partial_{x}^{3} U-\frac{b}{2} \partial_{x}^{2} \partial_{t}(U+N)-\frac{d}{2} \partial_{x}^{2} \partial_{t}(U-N)\right)=0 \\
\partial_{t} N-\partial_{x} N+\quad & \varepsilon\left(\frac{1}{8} \partial_{x}\left(U^{2}+3 N^{2}+2 U N\right)\right. \\
& \left.-a \partial_{x}^{3} N-\frac{b}{2} \partial_{x}^{2} \partial_{t}(U+N)+\frac{d}{2} \partial_{x}^{2} \partial_{t}(U-N)\right)=0
\end{aligned}\right.
$$

As usual for long wave BKW expansions, we seek approximate solutions $\left(U_{a}, N_{a}\right)$ of (5.41) under the form

$$
\begin{aligned}
& U_{a}(t, x)=U_{0}(\varepsilon t, x-t)+\varepsilon U_{1}(\varepsilon t, t, x), \\
& N_{a}(t, x)=N_{0}(\varepsilon t, x+t)+\varepsilon N_{1}(\varepsilon t, t, x), \\
& \left.\left(U_{a}, N_{a}\right)\right|_{t=0}=\left.(U, N)\right|_{t=0} .
\end{aligned}
$$

Plugging this ansatz into (5.41) and cancelling the first powers of $\varepsilon$ appearing in the expression thus obtained, one obtains thanks to the usual decoupling tools ([14], [4]) the following uncoupled equations for $U_{0}$ and $N_{0}$,

$$
\left\{\begin{array}{l}
\partial_{T} U_{0}+a \partial_{x}^{3} U_{0}-\frac{(b+d)}{2} \partial_{x}^{2} \partial_{t} U_{0}+\frac{3}{8} \partial_{x} U_{0}^{2}=0 \\
\partial_{T} N_{0}-a \partial_{x}^{3} N_{0}-\frac{(b+d)}{2} \partial_{x}^{2} \partial_{t} N_{0}+\frac{3}{8} \partial_{x} N_{0}^{2}=0
\end{array}\right.
$$

where $T$ stands for $\varepsilon t$. The equations determining the correctors $U_{1}$ and $N_{1}$ are

$$
\left\{\begin{array}{l}
\left(\partial_{t}+\partial_{x}\right) U_{1}=-\left(\frac{1}{8} \partial_{x}\left(N_{0}^{2}+2 U_{0} N_{0}\right)-\frac{(b-d)}{2} \partial_{x}^{2} \partial_{t} N_{0}\right) \\
\left(\partial_{t}-\partial_{x}\right) N_{1}=-\left(\frac{1}{8} \partial_{x}\left(U_{0}^{2}+2 U_{0} N_{0}\right)-\frac{(b-d)}{2} \partial_{x}^{2} \partial_{t} U_{0}\right)
\end{array}\right.
$$




\subsection{Estimates for the correctors}

Equations (5.44) give $\left(U_{1}, N_{1}\right)$ in terms of $\left(U_{0}, N_{0}\right)$. These equations can be solved explicitly:

$$
\begin{aligned}
U_{1}(T, t, x)= & -\frac{1}{16}\left(N_{0}^{2}(T, x+t)-N_{0}^{2}(T, x-t)\right) \\
& -\frac{(b-d)}{2}\left(\partial_{x} \partial_{t} N_{0}(T, x+t)-\partial_{x} \partial_{t} N_{0}(T, x-t)\right) \\
& -\frac{1}{4} \partial_{x} U_{0}(T, x-t) \int_{0}^{t} N_{0}(T, x-t+2 s) d s \\
& -\frac{1}{8} U_{0}(T, x-t)\left(N_{0}(T, x+t)-N_{0}(T, x-t)\right),
\end{aligned}
$$

and a similar expression holds for $N_{1}$. For all $s \in \mathbb{R}$, the terms which appear at the r.h.s. of $(5.45)$ are obviously bounded in $L^{\infty}\left(\left[0, T_{0}\right] \times \mathbb{R}_{t} ; H^{s}(\mathbb{R})\right)$ provided that $\left(U_{0}, N_{0}\right) \in L^{\infty}\left(\left[0, T_{0}\right] ; H^{\sigma}(\mathbb{R})\right)^{2}$ for some $\sigma$ big enough, except possibly the term

$$
W_{1}(T, t, x):=-\frac{1}{4} \partial_{x} U_{0}(T, x-t) \int_{0}^{t} N_{0}(T, x-t+2 s) d s .
$$

This latter term can be controlled thanks to the following lemma.

Lemma 5.1 Let $s \in \mathbb{N}$. Then there exists $\sigma$ big enough such that:

i. If $\left(U_{0}, N_{0}\right) \in L^{\infty}\left(\left[0, T_{0}\right] ; H^{\sigma}(\mathbb{R})\right)^{2}$ then $W_{1} \in L_{\text {loc }}^{\infty}\left(\left[0, T_{0}\right] \times \mathbb{R}_{t} ; H^{s}(\mathbb{R})\right)$ and

$$
\sup _{T \in\left[0, T_{0}\right]}\left|W_{1}(T, t, \cdot)\right|_{H^{s}(R)} \leq \text { Cst } \sqrt{t}, \quad \forall t \geq 0 ;
$$

i'. If moreover $N_{0}$ satisfies the following decay assumption: there exists $\alpha>$ $1 / 2$ such that

$$
\sup _{(T, x) \in\left[0, T_{0}\right] \times \mathbb{R}}\left|\left(1+x^{2}\right)^{\alpha} \partial_{x}^{\beta} N_{0}(T, x)\right|<\infty, \quad \beta=0, \ldots, s,
$$

then

$$
\sup _{T \in\left[0, T_{0}\right]}\left|W_{1}(T, t, \cdot)\right|_{H^{s}(R)} \leq \mathrm{Cst}, \quad \forall t \geq 0
$$

ii. In the periodic case, i.e. if $\left(U_{0}, N_{0}\right) \in L^{\infty}\left(\left[0, T_{0}\right] ; H^{\sigma}(\mathbb{T})\right)^{2}$ then

$$
W_{1}(T, t, x)=-\frac{t}{8 \pi} \partial_{x} U_{0}(T, x-t) \int_{0}^{2 \pi} N_{0}(T, x) d x+O(1) \text { as } t \rightarrow \infty .
$$

In particular, $W_{1}$ is bounded in $L^{\infty}\left(\left[0, T_{0}\right] ; H^{s}(\mathbb{T})\right)$ when $N_{0}(T, \cdot)$ has zero mean value for all $T \in\left[0, T_{0}\right]$. Otherwise, it grows secularly linearly in $t$.

Proof.

Point i. is classical (e.g. [15], [4]). We recall the proof in the case $s=0$. From (5.46), one deduces

$$
\begin{aligned}
\left|W_{1}(T, t, \cdot)\right|_{L^{2}(\mathbb{R})} & \leq \frac{1}{4}\left|\partial_{x} U_{0}(T)\right|_{L^{2}(\mathbb{R})}\left|\int_{0}^{t} N_{0}(T, x-t+2 s) d s\right|_{L^{\infty}(\mathbb{R})} \\
& \leq \operatorname{Cst} \sqrt{t}\left|\partial_{x} U_{0}(T)\right|_{L^{2}(\mathbb{R})}\left|N_{0}(T)\right|_{L^{2}(\mathbb{R})}
\end{aligned}
$$


which easily yields the desired estimate.

Point i'. is in the spirit of Lemma 5.5 of [19] and Prop. 3.5 of [15], and its proof is quite obvious in the present case. Finally, ii. is deduced easily from (5.46) by expanding $N_{0}$ into Fourier series.

\subsection{Validity of the uncoupled approximations for the diagonalized symmetric system}

We first consider the error made when approximating the exact solution $(U, N)$ of (5.41) with initial conditions $\left(U^{0}, N^{0}\right)$ by $\left(U_{a}, N_{a}\right)$ as given by (5.42)-(5.44).

Proposition 5.1 Let $s \in \mathbb{N}$. Then there exists $\sigma$ big enough and $T_{0}>0$ such that:

i. If $\left(U_{0}, N_{0}\right) \in L^{\infty}\left(\left[0, T_{0}\right] ; H^{\sigma}(\mathbb{R})\right)^{2}$ then

$$
\left|(U, N)-\left(U_{a}, N_{a}\right)\right|_{L^{\infty}\left([0, t], H^{s}(\mathbb{R})\right)^{2}} \leq \mathrm{Cst} \varepsilon^{2} t^{3 / 2}, \quad \forall t \in\left[0, \frac{T_{0}}{\varepsilon}\right] ;
$$

i'. If moreover $U_{0}$ and $N_{0}$ satisfy the following decay assumption: there exists $\alpha>1 / 2$ such that

$$
\sup _{(T, x) \in\left[0, T_{0}\right] \times \mathbb{R}}\left|\left(1+x^{2}\right)^{\alpha}\left(\partial_{x}^{\beta} U_{0}(T, x), \partial_{x}^{\beta} N_{0}(T, x)\right)\right|<\infty, \quad \beta=0, \ldots, s,
$$

then

$$
\left|(U, N)-\left(U_{a}, N_{a}\right)\right|_{L^{\infty}\left([0, t], H^{s}(\mathbb{R})\right)^{2}} \leq \mathrm{Cst} \varepsilon^{2} t, \quad \forall t \in\left[0, \frac{T_{0}}{\varepsilon}\right]
$$

ii. In the periodic case, i.e. if $\left(U_{0}, N_{0}\right) \in L^{\infty}\left(\left[0, T_{0}\right] ; H^{\sigma}(\mathbb{T})\right)^{2}$ then

$$
\left|(U, N)-\left(U_{a}, N_{a}\right)\right|_{L^{\infty}\left([0, t], H^{s}(\mathbb{R})\right)^{2}} \leq \mathrm{Cst} \varepsilon^{2} t^{2}, \quad \forall t \in\left[0, \frac{T_{0}}{\varepsilon}\right] ;
$$

ii'. If moreover the initial conditions $U^{0}$ and $V^{0}$ satisfy $\int_{0}^{2 \pi} U^{0}=\int_{0}^{2 \pi} N^{0}=0$ then

$$
\left|(U, N)-\left(U_{a}, N_{a}\right)\right|_{L^{\infty}\left([0, t], H^{s}(\mathbb{R})\right)^{2}} \leq \mathrm{Cst} \varepsilon^{2} t, \quad \forall t \in\left[0, \frac{T_{0}}{\varepsilon}\right] .
$$

\section{Proof.}

The approximate solution $\left(U_{a}, N_{a}\right)$ satisfies (5.41) with an error term of size $O\left(\varepsilon^{2} \sqrt{t}\right), O\left(\varepsilon^{2}\right), O\left(\varepsilon^{2} t\right)$ and $O\left(\varepsilon^{2}\right)$ in cases i., i'., ii. and ii'. respectively, by Lemma 5.1. Standard energy estimates on (5.41) therefore yield the results of the proposition.

Remark 5.1 In order to use Lemma 5.1 in case i'. (resp. ii'.), we must know that $U_{0}(T, \cdot)$ and $N_{0}(T, \cdot)$ satisfy the decay condition (resp. zero mean value condition) for all $T \in\left[0, T_{0}\right]$ and not only for the initial condion $\left(U^{0}, N^{0}\right)$. It is quite classical that these properties are propagated by the "KdV" equations (5.43) (see e.g. Prop. 6.3 of [19] for the propagation of the decay condition by the usual KdV equation). 
Proposition 5.2 In the periodic case, if the initial data $U^{0}(x)$ or $N^{0}(x)$ have nonzero mean value, there exist $T_{0} \geq T_{1}>0$ and $C>0$ such that for all $t \in\left[0 \frac{T_{1}}{\varepsilon}\right]$, one has

$$
\left|(U, N)-\left(U_{0}, N_{0}\right)\right|_{L^{\infty}\left([0, t], H^{s}(\mathbb{R})\right)^{2}} \geq C \varepsilon t .
$$

Remark 5.2 This means that in the periodic case, without the zero mean value condition, the KdV approximation is not valid on long time scales.

\section{Proof.}

The second term of the approximate solution $\left(U_{a}, N_{a}\right)$ has in this case a linear growth in time as shown by ii of Lemma 5.1. Therefore $U-U_{0}$ is bounded from below by $C_{1} \varepsilon t-C_{2} \varepsilon^{2} t^{2}$. The result follows.

\subsection{Validity of the uncoupled approximation for the Eu- ler equations}

In the previous sections, we derived a class a uncoupled $\mathrm{KdV}$-like equations (5.43) starting from systems (5.40) of the class $\Sigma$. We now derive a set of two uncoupled $\mathrm{KdV}$ equations starting from the original Boussinesq system (2.1), which reads, in $1 D$,

$$
\left\{\begin{array}{l}
\partial_{t} v+\partial_{x} \eta+\frac{\varepsilon}{2} \partial_{x} v^{2}=0 \\
\partial_{t} \eta+\partial_{x} v+\varepsilon\left(\partial_{x}(\eta v)+\frac{1}{3} \partial_{x}^{3} v\right)=0 .
\end{array}\right.
$$

Exactly as in Section 5.1, one can diagonalize this system introducing the unknowns $F=v+\eta$ and $G=v-\eta$, and look for an approximate solution $\left(F_{a}, G_{a}\right)$ of $(F, G)$ of the form

$$
\begin{aligned}
& F_{a}(t, x)=F_{0}(\varepsilon t, x-t)+\varepsilon F_{1}(\varepsilon t, t, x), \\
& G_{a}(t, x)=G_{0}(\varepsilon t, x+t)+\varepsilon G_{1}(\varepsilon t, t, x), \\
& \left.\left(F_{a}, G_{a}\right)\right|_{t=0}=\left.(F, G)\right|_{t=0} .
\end{aligned}
$$

It turns out that the uncoupled equations $F_{0}$ and $G_{0}$ must satisfy are exactly the same equations (5.43) as for the symmetric case, provided one takes $b=$ $d=0$ and $a=\frac{1}{6}$. Therefore, $\left(f^{\varepsilon}, g^{\varepsilon}\right)$ defined as

$$
f^{\varepsilon}(t, x)=F_{0}(\varepsilon t, x-t), \quad g^{\varepsilon}(t, x)=G_{0}(\varepsilon t, x+t),
$$

solve the set of equations

$$
\left\{\begin{array}{l}
\left(\partial_{t}+\partial_{x}\right) f^{\varepsilon}+\varepsilon\left(\frac{3}{8} \partial_{x} f^{\varepsilon 2}+\frac{1}{6} \partial_{x}^{3} f^{\varepsilon}\right)=0 \\
\left(\partial_{t}-\partial_{x}\right) g^{\varepsilon}+\varepsilon\left(\frac{3}{8} \partial_{x} g^{\varepsilon 2}-\frac{1}{6} \partial_{x}^{3} g^{\varepsilon}\right)=0
\end{array}\right.
$$


Construction of the $\mathrm{KdV}$ approximation for the water-waves equations. Consider initial data $\left(v_{0}, \eta_{0}\right)$ for the Euler equations (3.5)-(3.6) and denote by $\left(v^{\varepsilon}, \eta^{\varepsilon}\right)$ the associated family of solutions. The KdV approximation to $\left(v^{\varepsilon}, \eta^{\varepsilon}\right)$ is constructed as follows: let $f_{0}:=v_{0}+\eta_{0}$ and $g_{0}:=v_{0}-\eta_{0}$ and denote by $\left(f^{\varepsilon}, g^{\varepsilon}\right)$ the family of solution to (5.48) with initial condition $\left(f_{0}, g_{0}\right)$. The KdV approximation $\left(v_{K d V}^{\varepsilon}, \eta_{K d V}^{\varepsilon}\right)$ is then defined as $v_{K d V}^{\varepsilon}=\frac{f^{\varepsilon}+g^{\varepsilon}}{2}$, $\eta_{K d V}^{\varepsilon}=\frac{f^{\varepsilon}-g^{\varepsilon}}{2}$. For any $k \in \mathbb{N}$ and $s \in \mathbb{R}$, note that for sufficiently smooth initial data there exists $T_{0}>0$ such that both $\left(v^{\varepsilon}, \eta^{\varepsilon}\right)$ and $\left(v_{K d V}^{\varepsilon}, \eta_{K d V}^{\varepsilon}\right)$ are bounded families in $W^{k, \infty}\left(\left[0, \frac{T_{0}}{\varepsilon}\right], H^{s}(\mathbb{R})\right)$.

Choice of a reference symmetric system. As said above, if one choses $a=c=1 / 6$ and $b=d=0$ in (5.40) (which can be obtained by taking $\lambda=\mu=1$ and $\theta^{2}=2 / 3$ ), the asymptotic uncoupled equations (5.43) coincide with the uncoupled $\mathrm{KdV}$ equations satisfied by $F_{0}$ and $G_{0}$. It is therefore natural to consider the symmetric system $S_{\sqrt{2 / 3}, 1,1}^{\prime}$ which is given by (5.43) with this choice of parameters. From Th. 3.2 the approximation $\left(v_{a p p}^{\varepsilon}, \eta_{\text {app }}^{\varepsilon}\right)$ given by (3.8) and (3.9) (with $\underline{\lambda}=\underline{\mu}=1$ and $\underline{\theta}^{2}=2 / 3$ ) satisfies

$$
\left|\left(v^{\varepsilon}, \eta^{\varepsilon}\right)-\left(v_{a p p}^{\varepsilon}, \eta_{a p p}^{\varepsilon}\right)\right|_{L^{\infty}\left(0, t, H^{s}(\mathbb{R})\right)^{2}} \leq \operatorname{Cst} \varepsilon^{2} t, \quad t \in\left[0, \frac{T_{0}}{\varepsilon}\right] .
$$

Error estimate for the $\mathbf{K d V}$ approximation. We can now estimate the error made when approximating the solution $\left(v^{\varepsilon}, \eta^{\varepsilon}\right)$ of the Euler equations by the $\mathrm{KdV}$ approximation $\left(v_{K d V}^{\varepsilon}, \eta_{K d V}^{\varepsilon}\right)$ constructed above. One has

$$
\begin{aligned}
\left(v^{\varepsilon}, \eta^{\varepsilon}\right)-\left(v_{K d V}^{\varepsilon}, \eta_{K d V}^{\varepsilon}\right) & =\left(v^{\varepsilon}, \eta^{\varepsilon}\right)-\left(v_{a p p}^{\varepsilon}, \eta_{a p p}^{\varepsilon}\right)+\left(v_{a p p}^{\varepsilon}, \eta_{a p p}^{\varepsilon}\right)-\left(v_{K d V}^{\varepsilon}, \eta_{K d V}^{\varepsilon}\right) \\
& =O\left(\varepsilon^{2} t\right)+\left(v_{a p p}^{\varepsilon}, \eta_{a p p}^{\varepsilon}\right)-\left(v_{K d V}^{\varepsilon}, \eta_{K d V}^{\varepsilon}\right),
\end{aligned}
$$

according to (5.49). Inverting approximatively the nonlinear pseudodifferential change of variables, one can observe that

$\left|\left(v_{a p p}^{\varepsilon}, \eta_{a p p}^{\varepsilon}\right)-\left(v_{K d V}^{\varepsilon}, \eta_{K d V}^{\varepsilon}\right)\right|_{L^{\infty}\left(0, t, H^{s}(\mathbb{R})\right)^{2}}=\left|\left(v_{\Sigma}^{\varepsilon}, \eta_{\Sigma}^{\varepsilon}\right)-\left(\widetilde{v_{K d V}^{\varepsilon}}, \widetilde{\eta_{K d V}^{\varepsilon}}\right)\right|_{L^{\infty}\left(0, t, H^{s}(\mathbb{R})\right)^{2}}+O\left(\varepsilon^{2}\right)$,

with

$$
\widetilde{v_{K d V}^{\varepsilon}}=\left(1-\frac{\varepsilon}{6} \partial_{x}^{2}\right)^{-1} v_{K d V}^{\varepsilon}\left(1+\frac{\varepsilon}{2} \eta_{K d V}^{\varepsilon}\right), \quad \widetilde{\eta_{K d V}^{\varepsilon}}=\eta_{K d V}^{\varepsilon},
$$

and where $\left(v_{\Sigma}^{\varepsilon}, \eta_{\Sigma}^{\varepsilon}\right)$ denotes the solution to the symmetric system $S_{\sqrt{2 / 3,1,1}}^{\prime}$ with initial conditions (3.8). In accordance with the notations of Section 5.1, write $U=v_{\Sigma}^{\varepsilon}+\eta_{\Sigma}^{\varepsilon}$ and $N=v_{\Sigma}^{\varepsilon}-\eta_{\Sigma}^{\varepsilon}$ so that $\left(U_{a}, N_{a}\right)$ as constructed in Section 5.1 gives a good asymptotic description of $(U, N)$.

Now remark that $\widetilde{F}:=\widetilde{v_{K d V}^{\varepsilon}}+\widetilde{\eta_{K d V}^{\varepsilon}}$ and $\widetilde{G}:=\widetilde{v_{K d V}^{\varepsilon}}-\widetilde{\eta_{K d V}^{\varepsilon}}$ solve the uncoupled $\mathrm{KdV}$ equations (5.43) up to a $O\left(\varepsilon^{2}\right)$ term. It follows that if one replaces $\left(U_{0}, N_{0}\right)$ by $(\widetilde{F}, \widetilde{G})$ in the ansatz $(5.42)$, the results of Prop. 5.1 are not altered. 
It follows from these points that the error made by the $\mathrm{KdV}$ approximation can be evaluated in $L^{\infty}\left(0, t, H^{s}(\mathbb{R})\right)$ norm as

$$
\left\|\left(v^{\varepsilon}, \eta^{\varepsilon}\right)-\left(v_{K d V}^{\varepsilon}, \eta_{K d V}^{\varepsilon}\right)\right\|=O\left(\varepsilon^{2} t\right)+\left\|(U, N)-\left(U_{a}, N_{a}\right)\right\| .
$$

Our final result is now a simple consequence of Prop. 5.1:

Theorem 5.1 Let $s \in \mathbb{R}$. There exists $\sigma$ large enough such that if $\left(v_{0}, \eta_{0}\right) \in$ $\left(H^{\sigma}(\mathbb{R})\right)^{2}$ there exists $T_{0}>0$ such that:

i.

$$
\left|\left(v^{\varepsilon}, \eta^{\varepsilon}\right)-\left(v_{K d V}^{\varepsilon}, \eta_{K d V}^{\varepsilon}\right)\right|_{L^{\infty}\left([0, t], H^{s}(\mathbb{R})\right)^{2}} \leq \mathrm{Cst} \varepsilon^{2} t^{3 / 2}, \quad \forall t \in\left[0, \frac{T_{0}}{\varepsilon}\right]
$$

i'. If moreover $v_{0}$ and $\eta_{0}$ satisfy the following decay assumption: there exists $\alpha>1 / 2$ such that

$$
\sup _{x \in \mathbb{R}}\left|\left(1+x^{2}\right)^{\alpha}\left(\partial_{x}^{\beta} v_{0}(x), \partial_{x}^{\beta} \eta_{0}(x)\right)\right|<\infty, \quad \beta=0, \ldots, s,
$$

then

$$
\left|\left(v^{\varepsilon}, \eta^{\varepsilon}\right)-\left(v_{K d V}^{\varepsilon}, \eta_{K d V}^{\varepsilon}\right)\right|_{L^{\infty}\left([0, t], H^{s}(\mathbb{R})\right)^{2}} \leq \mathrm{Cst} \varepsilon^{2} t, \quad \forall t \in\left[0, \frac{T_{0}}{\varepsilon}\right]
$$

ii. In the periodic case, i.e. if $\left(v_{0}, \eta_{0}\right) \in H^{\sigma}(\mathbb{T})^{2}$ then

$$
\left|\left(v^{\varepsilon}, \eta^{\varepsilon}\right)-\left(v_{K d V}^{\varepsilon}, \eta_{K d V}^{\varepsilon}\right)\right|_{L^{\infty}\left([0, t], H^{s}(\mathbb{T})\right)^{2}} \leq \mathrm{Cst} \varepsilon^{2} t^{2}, \quad \forall t \in\left[0, \frac{T_{0}}{\varepsilon}\right] ;
$$

ii'. If moreover $\int_{0}^{2 \pi} v_{0}=\int_{0}^{2 \pi} \eta_{0}=0$ then

$$
\left|\left(v^{\varepsilon}, \eta^{\varepsilon}\right)-\left(v_{K d V}^{\varepsilon}, \eta_{K d V}^{\varepsilon}\right)\right|_{L^{\infty}\left([0, t], H^{s}(\mathbb{T})\right)^{2}} \leq \mathrm{Cst} \varepsilon^{2} t, \quad \forall t \in\left[0, \frac{T_{0}}{\varepsilon}\right] .
$$

iii. In the periodic case, if $v_{0}$ or $\eta_{0}$ have nonzero mean value, there exist $T_{0} \geq T_{1}>0$ and $C>0$ such that for all $t \in\left[0 \frac{T_{1}}{\varepsilon}\right]$, one has

$$
\left|\left(v^{\varepsilon}, \eta^{\varepsilon}\right)-\left(v_{K d V}^{\varepsilon}, \eta_{K d V}^{\varepsilon}\right)\right|_{L^{\infty}\left([0, t], H^{s}(\mathbb{T})\right)^{2}} \geq C \varepsilon t .
$$

Remark 5.3 i. In [19], it is proved that under the decay assumption of $\mathbf{i}$, the $K d V$ approximation furnishes an approximate solution with an error estimate of size $O\left(\varepsilon^{1 / 4}\right)$. The error estimates $\mathbf{i}$ ' and even $\mathbf{i}$ improve that of [19]. In case i', our estimate is sharp (since we have constructed the following term in the expansion). In the periodic case, our results show that the uncoupled $K d V$ approximation diverges from the exact solution of the Euler equation unless the non-realistic zero-mean-value assumption on the initial data is made.

ii. No existence result exists in the periodic framework, so that as for the $3 D$-case, we assume the existence of a family of solutions $\left(v^{\varepsilon}, \eta^{\varepsilon}\right)$ to the Euler equations, over times $O(1 / \varepsilon)$ and with initial condition $\left(v_{0}, \eta_{0}\right)$. 


\section{References}

[1] A. A. Alazman, J. P. Albert, J. L. Bona, M. Chen and J. Wu. Comparison between the BBM equation and a Boussinesq system. Preprint: 2003.

[2] J. P. Albert and J. L. Bona. Comparisons between model equations for long waves. J. Nonlinear Sci., 1:345-374, 1991.

[3] T. B. Benjamin, J. L. Bona, and J. J. Mahony. Model equations for long waves in nonlinear dispersive systems. Philos. Trans. Roy. Soc. London Ser. A, 272:47-78, 1972.

[4] W. Ben Youssef and T. Colin. Rigorous derivation of Korteweg-de Vriestype systems from a general class of nonlinear hyperbolic systems. M2AN Math. Model. Numer. Anal., 34(4):873-911, 2000.

[5] J. L. Bona, M. Chen, and J.-C. Saut. Boussinesq equations and other systems for small-amplitude long waves in nonlinear dispersive media. I. Derivation and linear theory. J. Nonlinear Sci., 12(4):283-318, 2002.

[6] J. L. Bona, M. Chen, and J.-C. Saut. Boussinesq equations and other systems for small-amplitude long waves in nonlinear dispersive media. II. Nonlinear theory. Preprint: 2003.

[7] J. L. Bona, W. G. Pritchard and L. R. Scott. An evaluation of a model equation for water waves. Philos. Trans. Royal Soc. London, Series A, 302: 457-510, 1981.

[8] J. L. Bona, W. G. Pritchard and L. R. Scott. A comparison of solutions of two model equations for long waves. In Fluid Dynamics in Astrophysics and Geophysics (ed. N. Lebovitz), vol. 20 of Lectures in Appl. Math., American Math. Soc., Providence, R.I.: 235-267, 1983.

[9] J. L. Bona and R. Smith. A model for the two-way propagation of water waves in a channel. Math. Proc. Cambridge Philos. Soc, 79: 167-182, 1976.

[10] M. J. Boussinesq. Théorie de l'intumescence liquide appelée onde solitaire ou de translation se propageant dans un canal rectangulaire. C. R. Acad. Sci. Paris Sér. A-B, 72:755-759, 1871.

[11] W. Craig. An existence theory for water waves and the Boussinesq and Korteweg-de Vries scaling limits. Comm. Partial Differential Equations, 10(8):787-1003, 1985.

[12] W. Craig, U. Schantz, and C. Sulem. The modulational regime of threedimensional water waves and the Davey-Stewartson system. Ann. Inst. H. Poincaré Anal. Non Linéaire, 14(5):615-667, 1997. 
[13] W. Craig, C. Sulem, and P.-L. Sulem. Nonlinear modulation of gravity waves: a rigorous approach. Nonlinearity, 5(2):497-522, 1992.

[14] D. Lannes. Dispersive effects for nonlinear geometrical optics with rectification. Asymptot. Anal, 18 (1998), no. 1-2, 111-146.

[15] D. Lannes. Secular growth estimates for hyperbolic systems. J. Differential Equations, 190 (2003), no. 2, 466-503.

[16] D. Lannes. Well-posedness of the water-waves equations. Preprint Université Bordeaux 1.

[17] V. I. Nalimov. The Cauchy-Poisson problem. Dinamika Splošn. Sredy, (Vyp. 18 Dinamika Zidkost. so Svobod. Granicami):104-210, 254, 1974.

[18] David P. Nicholls and Fernando Reitich. A new approach to analyticity of Dirichlet-Neumann operators. Proc. Roy. Soc. Edinburgh Sect. A, 131(6):1411-1433, 2001.

[19] G. Schneider and C. E. Wayne. The long-wave limit for the water wave problem. I. The case of zero surface tension. Comm. Pure Appl. Math., 53(12):1475-1535, 2000.

[20] C. E. Wayne and J. D. Wright. Higher order corrections to the KdV approximation for a Boussinesq equation. SIAM J. Appl. Dyn. Syst, 1 (2002), no. 2, 271-302.

[21] S. Wu. Well-posedness in Sobolev spaces of the full water wave problem in 2-D. Invent. Math., 130(1):39-72, 1997.

[22] S. Wu. Well-posedness in Sobolev spaces of the full water wave problem in 3-D. J. Amer. Math. Soc., 12(2):445-495, 1999. 